\title{
机器学习在有机化学中的应用
}

\author{
刘伊迪 ${ }^{\dagger}$ 杨骐 ${ }^{\dagger}$ 李遥张龙* 罗三中* \\ (清华大学化学系 基础分子科学中心 北京 100084)
}

\begin{abstract}
摘要 近年来, 由于计算能力、大数据和算法的不断进步, 人工智能(Artificial intelligence, AI)重新兴起, 已成为诸多研 究领域变革性发展背后的重要推动力. 机器学习(Machine learning, ML)是人工智能一个重要的研究领域. 随着化学信 息学的发展, 机器学习在化学领域展现出巨大的发展潜力, 也为有机化学的发展带来了新的机遇. 为帮助有机化学家 了解这一新兴领域, 对如何将机器学习策略应用于有机化学研究做简单介绍, 同时, 概括总结了机器学习在化合物性 质预测、分子从头设计、化学反应预测、逆合成分析和智能合成机器方面的应用实例, 分析讨论了当前机器学习在有 机化学领域面临的挑战和难题.
\end{abstract}

关键词 机器学习; 分子描述符; 算法; 化学性质预测; 分子从头设计; 化学反应预测; 逆合成分析

\section{Application of Machine Learning in Organic Chemistry}

\author{
Liu, Yidi ${ }^{\dagger} \quad$ Yang, $\mathrm{Qi}^{\dagger} \quad$ Li, Yao $\quad$ Zhang, Long* ${ }^{*} \quad$ Luo, Sanzhong* \\ (Center for Basic Molecular Science, Department of Chemistry, Tsinghua University, Beijing 100084)
}

\begin{abstract}
Driven by nowadays' computing power, big data technology as well as learning algorithm, artificial intelligence (AI) has gained trenmendous attentions and become a transformative approach in many research areas. One of the most extensively explored AI approaches in chemistry is (deep) machine learning, which provides new twists in the fields of organic chemistry. The workflow of machine learning (ML) study in organic chemistry is briefly introduced. Meanwhile, the application of ML in the accurate prediction of chemical properties, molecular de novo design, chemical reaction prediction, retrosynthetic analysis and artificial intelligence synthetic machine are also summarized. In the end, the current challenges in this field are analyzed and discussed.
\end{abstract}

Keywords machine learning; molecular descriptor; algorithm; chemical property prediction; de novo design; chemical reaction prediction; retrosynthesis analysis

创造拥有人类智慧的机器是人类自古以来的梦想, 从中国周朝的偃师造人到古希腊神话中的黄金机器人, 人类一直在探索生命和智慧背后的秘密. 伴随着计算机 基础理论和技术的诞生和快速发展, 这一古老梦想正逐 渐成为现实. 1956 年, 麦卡锡 ${ }^{[1]}$ 首次提出了人工智能 (Artificial intelligence, AI)的概念, 开启了这一全新的研 究领域. 半个多世纪以来, 伴随着计算机计算能力的快 速提升、大数据技术的发展以及学术界和工业界的通力 合作, 人工智能领域取得了令人瞩目的进展 ${ }^{[2]} .2016$ 年, 阿尔法狗(AlphaGo) ${ }^{[3]}$ 打败围棋世界冠军李世石这一历
史性时刻标志着人工智能走向了一个全新的高度, 其在 各个领域的应用也开始进入公众视野. 特别是最近十几 年开始兴起的机器学习和深度学习等相关领域的爆发 式进展, 在自然语言处理、图像识别、自动驾驶等领域, 已经大有赶超人类现有认知水平的势头 ${ }^{[4]}$.

机器学习(Machine learning)作为人工智能研究领域 的重要分支, 近年来得到飞速发展和广泛应用. 1959 年 Samuel 等 ${ }^{[5]}$ 首次提出机器学习的概念, 将其定义为在不 进行特定编程情况下给予计算机学习的能力 (Give computers the ability to learn without being explicitly pro-

\footnotetext{
* Corresponding authors. E-mail: luosz@tsinghua.edu.cn; zhanglong@tsinghua.edu.cn Received June 24, 2020; revised July 22, 2020; published online August 5, 2020 Dedicated to the 40th anniversary of Chinese Journal of Organic Chemistry. Project supported by the National Science \& Technology Fundamental Resource Investigation Program of China (No. 2018FY201200), the Tsinghua University Initiative Scientific Research Program (No. 2019Z07L01005) and the Natural Science Foundation of China (Nos. 22031006, 21672217, 21933008). 科技部基础资源调查项目(No. 2018FY201200)、清华大学理科双创项目(No. 2019Z07L01005)和国家自然科学基金(Nos. 22031006, 21672217, 21933008) 资助项目.

†共同第一作者(These authors contributed equally to this work).
} 
grammed). 如图 1 所示, 机器和人类的学习模式不乏相 通之处 ${ }^{[6]}$. 人类的大脑通过阅读、观察、探索和学习周 围世界的规则并获得各种技能, 比如语言、决策、游戏 等. 机器则通过海量数据和程序的自我学习, 获得类似 人类的语言、决策、游戏等各种能力. 机器获得学习能 力的前提是待解决问题存在基准真值(Ground truth), 通 过建立一个假设空间, 程序学习数据中的通用模式, 建 立模型并尝试预测基准真值. 预测值与基准真值越接 近, 模型越精确. 程序通过这样一个不断学习与优化的 过程, 试图获得人类大脑具有的学习和解决问题的能 力.

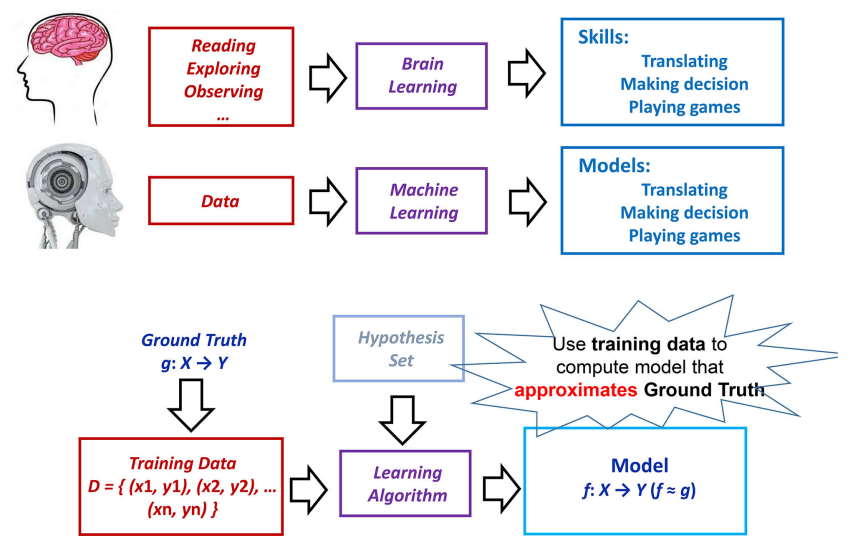

图 1 人类与机器的学习模式

Figure 1 Learning mode of human and machine

随着化学信息学的发展, 机器学习在化学领域已展 现出巨大的应用潜力, 在量子化学 ${ }^{[7]}$ 、密度泛函理论 (DFT) 计算 ${ }^{[8]}$ 、药物发现 ${ }^{[9]}$ 、分子从头设计 ${ }^{[10]}$ 、反应预测 与逆合成分析 ${ }^{[11]}$ 以及自动化合成 ${ }^{[12]}$ 等领域均已有重要 应用. 传统的有机化学研究过程中, 物理有机和量子化 学提供了强大的理论工具, 但面对复杂体系预测性有 限, 大多数新发现仍高度依赖于 “试错(Trio-errors)” 和 “偶缘(Serendipity)”. 而人工智能和机器学习以其强大 的学习能力、迭代能力、容易实现和易于部署的特性, 为 有机化学研究范式的革新带来了曙光. 本文主要综述机 器学习在有机化学研究中的应用, 简要介绍机器学习的 研究范式和在有机化学领域的应用模式, 结合最新的研 究进展展示具体的应用实例, 并总结和展望当前研究的 挑战和难题. 本文面向有机化学多个研究领域, 兼顾基 础性和前沿研究进展, 对涉及机器学习的诸多方面比如 数据、算法、描述符以及工作流程做基本介绍.

\section{1 机器学习如何应用于有机化学}

机器学习应用于有机化学一般遵循以下步骤(图 2): (1)数据集: 通过收集公开发表的文献、数据库、实验室 原始数据等方式汇总特定任务的数据集; (2)分子描述:
将化学分子和反应式转化为算法能够识别的形式; (3)模 型建立：选择针对特定问题的模型，选择合适的算法， 使用训练集对模型进行训练, 并用验证集对模型表现进 行评价; (4)模型应用: 使用训练好的模型预测未知结果; (5)讨论与分析：对预测结果(如物理化学特性或者反应活 性等)进行归因与解释. 本文重点介绍数据集、分子描述 符和算法, 并结合具体实例介绍模型训练、应用和分析.

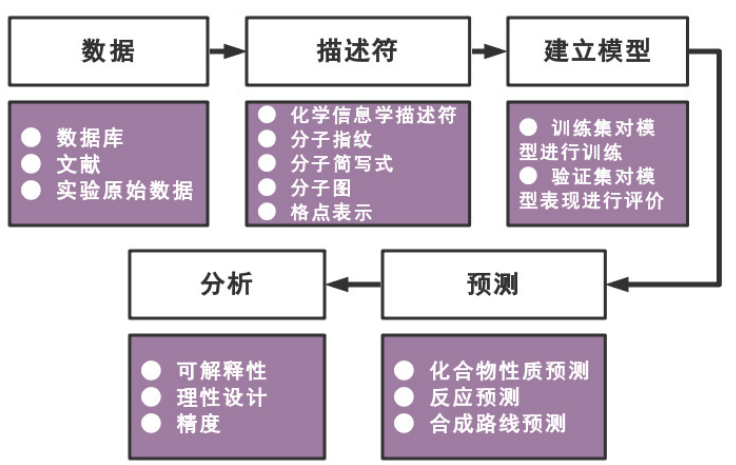

图 2 机器学习应用于有机化学的工作流程

Figure 2 Workflow of applying machine learning in organic chemistry

\section{1 数据集}

公开发表在杂志、专利中的数据需要通过提取、汇 总、整理才能成为可用的数据集(比如 SciFinder 和 Reaxys 等 ${ }^{[13]}$. 但通常来说，一般使用者并不能直接批 量获取这些数据. 最近, 一些课题组与 Reaxys 合作, 通 过其拥有的海量数据开展化学反应预测和逆合成分析 工作 ${ }^{[11]}$. 当前也有一些公开的化学数据库, 如 GDB- $13^{[14]}$ 及其子库 $\mathrm{QM} 7 / \mathrm{QM} 7 \mathrm{~b} 、 \mathrm{GDB}-17^{[15]}$ 及其子库 QM8、QM9 等, 收集了大量小分子化合物的量化信息. $\mathrm{ESOL}^{[16]}$ 和 FreeSolv ${ }^{[17]}$ 则收集了大量小分子化合物的物 理化学信息, 如水溶性和自由能信息. ZINC 数据库 ${ }^{[18]}$ 收 录了大量市售化合物的 3D 结构、供应商等信息, 可用 于虚拟篮选. 美国专利及商标局(United States Patent and Trademark Office, USPTO)提供了数十年间专利反应 数据, 并由 Lowe 等 ${ }^{[19]}$ 整理成了专门的反应数据集可供 下载使用.

数据的另一种获取来源是电子实验记录本. 许多药 物和化学相关公司运用电子实验记录本记录数据, 但相 关数据主要用于数据存储和知识产权目的, 通常难以直 接进行数据挖掘, 需要开放访问并且提供专门的化学信 息学环境 ${ }^{[20]}$. NextMove Software 开发的 HazELNut 工具 包可以从电子实验记录本系统中提取、格式转换和储存 实验数据, 并添加反应分类等附加的标注 ${ }^{[21]} .2013$ 年, 
罗氏与 Elsevier、NextMove 合作，整合公司内部的实验 数据和 Elsevier 的文献数据, 罗氏研究人员可以使用 Reaxys 的定制界面搜索和汶览所有的反应数据. 2014 年, 阿斯利康提取了电子实验记录本的数据并将其加载 到数据中心，支持网页搜索和其他外部应用程序. 除了 商业软件工具, 还有用于基本反应分析的开源软件, 例 如反应解码器(Reaction decoder tool, RDT)工具包, 能够 快速从化学反应中提取特征关系 ${ }^{[22]}$.

另一方面, 发表的文献和专利大多为最优条件下的 最好结果, 而失败的反应和被证明行不通的反应路线都 没有发表, 这将造成严重的数据偏误. 而电子实验记录 本不仅记录了反应中较优的结果, 也记录了较差的甚至 失败的实验结果, 不存在负面样本偏误. 因此, 电子实 验记录本记录的结果更加适用于实验数据挖掘以及预 测模型的建立和训练. 但即使已有数百万高质量的实验 结果收录在案，面对巨大的化学反应空间(反应物、产 物、反应条件等参数), 这些数据在空间中的分布相对来 说仍然十分稀疏 ${ }^{[23]}$. 近年来, 随着微流控和自动化技术 的不断进步, 高通量篎选装置能在较短时间里产生大量 优质的反应数据, 使系统探索更大范围反应空间成为可 能 ${ }^{[24]}$, 这些研究推动了有机合成的大数据化, 有望为智 能化学合成提供数据基础 ${ }^{[25]}$.

\section{2 分子描述}

数据收集完成后, 另一个关键的问题就是如何把分 子式转化为计算机能够识别的形式. 通过何种方式描述 化合物分子以及描述符是否包含了化合物分子中暗含 的关键信息, 将直接决定模型的预测效果. 化合物分子 描述方式种类繁多, 比较常见的有以下几种方式(图 3).

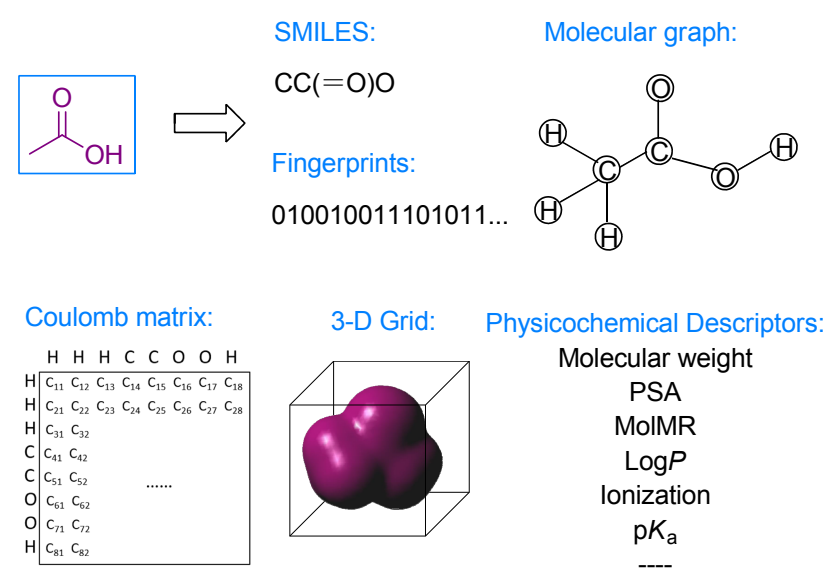

图 3 以乙酸示例六种常用的分子描述符

Figure 3 Molecular descriptors of acetic acid

\subsection{1 物理化学描述符}

这种方法通过提取分子中各种物理化学参数, 比如 $\log P 、 \mathrm{p} K_{\mathrm{a}}$ 和分子量(Molecular weight)等, 这些参数最终
汇总为一组特征向量并作为输入训练机器学习模型. 当 前常用的化学信息学软件, 如 ChemAxon ${ }^{[26] 、 R D K i t}{ }^{[27]}$ 和 $\mathrm{CDK}^{[28]}$ 等都可以快速方便地提取分子的特征. 此外, 通过半经验或 DFT 理论计算获得高精度分子结构特征 和理化参数也是较为常用的描述符生成方法.

\subsection{2 分子指纹}

这种方法将化合物分子转化为一串二进制向量，这 些二进制向量特征性地表示了某类分子碎片是否存在， 如同指纹一样描述了某一分子与其他分子的相似与不 同之处. 这里列举三种常见的分子指纹: (1)基于亚结构 的分子描述子 MACCS $^{[29]}$ 和 PubChem fingerprints ${ }^{[30]}$; (2) 基于拓扑和路径的指纹, 如 Daylight fingerprint 和 Tree

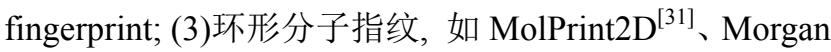
fingerprint ${ }^{[32]}$ 以及类似的 Extended-Connectivity Fingerprints (ECFP) ${ }^{[33]}$ 、Functional-Class Fingerprints(FCFP)) 等. 一些免费的开源化学信息学工具包支持对分子指纹的 提取，例如 RDKit、Open Babel ${ }^{[34]} 、 \mathrm{CDK}$ 和 Indigo ${ }^{[35]}$ 等.

\subsection{3 分子简写式}

最常用的分子简写式是简化分子线性输入规范 ${ }^{[36]}$ (Simplified molecular input line entry specification, SMILES)和国际化学标识符 ${ }^{[37]}$ ((International chemical Identifier, InChI), 标准化的化学标识格式可以解决数据 库中分子的非标准化命名问题. SMILES 利用 ASCII 字 符串，通过简单的原子符号、键符号和语言规则可以描 述分子的三维结构和化学反应. SMILES 的一种扩展格 式 SMARTS ${ }^{[38]}$ (SMILES arbitrary target specification)是 一种精确的子结构特征识别和原子分类的符号. InChI 能够唯一性地描述化学分子 ${ }^{[39]}$, 而 SMILES 不是唯一性 的.

\section{2 .4 分子图}

分子图法是将分子抽象为一张向量图或者无向图, 其中的原子和化学键抽象表达为图中的节点和边. 库伦 矩阵(Coulomb matrix ${ }^{[7,40]}$ ) 是分子图的另一种表示方法, 包含原子核排斥和自由原子的势能信息，并且对分子的 平移和旋转保持不变, Rupp 等 ${ }^{[7]}$ 将这一方法用于分子 原子化能预测, 精度能达到 $12.54 \mathrm{~kJ} / \mathrm{mol}$.

\subsection{5 格点(Grid)表示法}

格点表示法是将分子放置于二维或三维网格中，通 过每个网格的位置和包含的分子信息进行编码. 其中三 维格点方法可以提取分子的空间结构特征，这些策略已 经被用于蛋白质配体打分模型 ${ }^{[41]}$ 和立体选择性预测模 型 ${ }^{[42]}$ 等工作中.

\section{3 机器学习算法}

算法是人工智能的核心, 目前常用的机器学习算法 
种类繁多. 从学习方式来分, 可以分为监督学习 (Supervised learning)、无监督学习(Unsupervised learning)、半监督学习(Semi-supervised learning)和强化学习 (Reinforcement learning ${ }^{[43]}$ )等. 从机器学习的算法基础 来分, 则可以分为基于统计学的传统机器学习算法和神 经网络算法. 目前常用的传统机器学习算法主要包括贝 叶斯方法(Bayesian ${ }^{[44]}$ )、决策树(Decision tree $\mathrm{e}^{[45]}$ )及其衍 生方法、朴素贝叶斯(Naive Bayes $\left.{ }^{[46]}\right)$ 、支持向量机 (Support vector machine ${ }^{[47]}$ )、聚类方法(Cluster analysis ${ }^{[48]}$ )、集成学习算法(Ensemble methods, 如著名的基于 树的随机森林方法, Random forest $\left.{ }^{[49]}\right)$ 等, 这类方法被广 泛应用于包括有机化学在内的各个领域. 相关算法原理 可参考相关综述及统计学相关书籍 ${ }^{[50]}$, 在此不做过多 介绍. Scikit-learn ${ }^{[51]}$ Python 工具库囊括了大部分的经典 机器学习算法, 可以结合 RDkit 等化学信息学工具直接 调用，使用非常方便.

另一类则是基于大脑神经元(Brain-inspired)的工作 原理发展起来的神经网络模型. 与创造 “人工大脑” 不 同, 这些程序试图理解大脑是如何工作的. 这类模型主 要有两个分支. 一类被称为脉冲神经网络(Spike neural network ${ }^{[52]}$ ), 该网络基于拟合生物神经元发射和接收脉 冲信号的机制. 另一类则是现在极为流行的神经网络 (Neural network $)^{[53]}$. 通过神经元的多层级连接, 神经网 络能够提取输入中的关键特征, 并提取出这些特征与输 出之间复杂的关系. 随着计算机运算能力的爆炸性增 长, 越来越复杂的神经网络, 即深度神经网络也开始被 应用在化学研究的各个领域, 并展现出巨大的潜力. 深 度神经网络可以使用更多神经元节点和更深的网络层, 因此其特征抽象能力也大大提高. 同时, 神经网络结构 和算法也飞速发展, 比如通过 Dropout ${ }^{[54]}$ 和 DropConnect ${ }^{[55]}$ 方法解决过拟合问题, 通过 Rectified linear unit (ReLU) $)^{[56]}$ 解决梯度消失问题, 通过图神经网 络 ${ }^{[57]}$ 解决分子拓扑结构表示的问题 ${ }^{[8]}$ 等. 目前流行的 深度神经网络主要包含全连接神经网络(Full connected neural network, FCNN)、卷积神经网络(Convolutional neural network, CNN)、循环神经网络(Recurrent Neural Network, RNN)、自动编码器(Auto encoder, AE)、生成对 抗网络(Generative adversarial network, GAN)及其相关 变种. 关于神经网络的工作原理目前已有大量书籍文 献, 在此不做过多介绍. 大部分机器学习框架都是开源 的, 如 TensorFlow、PyTorch、GLUON、Caffe、Theano 等框架已经被广泛应用于各个方面.

对于有机化学工作者来说, 最关心的问题是如何选 择已有的算法来解决面临的有机化学问题. 从学习方式 来看, 目前有机化学领域主要采用监督学习算法, 即利
用已标记的有限化学数据集, 通过某种学习策略/方法 建立模型, 实现对新数据/实例的标记(分类)或映射(回 归). 监督学习要求训练样本的标签(如物化性质、药理 活性、反应选择性等)已知, 标签的精确度越高, 样本越 具有代表性, 学习模型的准确度越高. 此外, 很多有机 化学问题并不符合相似性原则, 结构的微小变化就可能 造成药物活性、催化活性等性质的巨大变化, 非基于距 离的算法(Non-distance-based algorithms)比如随机森林、 神经网络等对这类问题更加合适 ${ }^{\left[{ }^{[9]}\right.}$. 在具体的研究中, 往往可通过对已有算法进行篮选和验证来确定最适用 的机器学习算法. 需要注意的是, 基于训练集和验证集 得到的最优模型并不一定是最适用的模型, 它可能存在 过拟合或者泛化能力差等问题, 一般还需要经过外部测 试集的进一步检验来确定最优的预测模型.

\section{2 机器学习在有机化学领域的应用}

机器学习在有机化学领域最早的工作可追溯到 20 世纪 60 年代, Corey 等 ${ }^{[60]}$ 发展了基于规则的计算机辅助 合成设计程序 (Computer-aided synthetic planning, CASP). CASP 的目标是辅助化学家快速高效地实现小 分子化合物的合成. 其以分子结构作为输入, 输出一系 列详细的反应方案, 每个方案通过一系列可行的反应步 骤将目标连接到可购买的起始原料. 20 世纪 60 年代至 90 年代, 受到计算资源的限制, 早期工作主要依赖于专 家编码的反应规则和启发式策略, 通过给定的判断规则 给出可能的化学键断裂和生成的位置. 在过去的 20 年 间, 随着大规模化学数据库的发展, 数以百万计的反应 数据可以应用于机器学习模型的训练和验证. 同时, 神 经网络与深度学习算法的快速发展也推动了机器学习 在化合物性质预测、分子从头设计、化学反应预测、逆 合成分析以及智能合成机器等领域的应用 ${ }^{[61]}$.

\section{1 化合物性质预测}

机器学习很早就已应用于化合物性质预测. 近年 来, 深度神经网络在该领域的应用展现出了巨大的优 势, 并频繁出现在各种化合物性质与活性预测挑战赛 中. 2015 年, Dahl 等 ${ }^{[62]}$ 使用 $2 \mathrm{D}$ 拓扑描述符作为输入, 将 深度神经网络方法应用于 Merck Kaggle 挑战赛. 在 15 个化合物活性预测中有 13 个目标都显著高于当时最优 的预测结果. 这一方法有几个显著特点: (1)深度神经网 络可以同时处理数千个描述符而无需特征选择; (2) Dropout 可以避免传统神经网络过拟合的问题; (3)超参 优化可以最大限度地提升深度神经网络的预测效果; (4) 多任务深度神经网络的预测效果明显好于单任务神经 网络. 2016 年, Mayr 等 ${ }^{[63]}$ 运用多任务神经网络赢得了 Tox21 挑战赛, 他们选用多种描述符(3D/2D 描述符、预 
先定义的药效基团)和 ECFP 分子指纹输入模型对 12000 种化合物的 12 种毒性指标进行了建模, 与 Dahl 等的神 经网络类似, 他们也将 Dropout 方法和 ReLu激活函数应 用于深度神经网络, 并使用 GPU 并行计算加速模型训 练. 研究结果表明深度神经网络具备很强的特征提取能 力, 模型可以有效识别并提取出各种可能的毒性基团特 征. 随后的多项对比研究均发现, 相较于传统机器学习 算法, 神经网络算法在化合物生物活性预测方面表现出 较优的预测能力 ${ }^{[64]}$. 目前, 神经网络算法在药物生理活 性、毒性、溶解度等性质预测方面均实现了较好的应 用 ${ }^{[65]}$. 需要指出的是, 深度神经网络的表现很大程度上 取决于训练数据规模和质量, 数据不足或质量不高可能 很难得到稳健且迁移能力较好的模型. 最近, Kim 等 ${ }^{[66]}$ 利用贝叶斯推理进行定量的不确定性分析来提高预测 的可靠性, 他们选择分子图作为模型输入, 采用贝叶斯 图卷积网络(Bayesian graph convolutional network)对化 合物生物活性和毒性进行分类建模和预测, 并可同时定 量给出引起不确定性的因素, 有效提高了 ChEMBL 数 据集中分子表皮生长因子受体(Epidermal growth factor receptor, EGFR)抑制活性预测的准确性 ${ }^{[67]}$.

机器学习在有机化合物物理化学性质预测方面也 表现出不俗的潜力, 以有机物 $\mathrm{p} K_{\mathrm{a}}$ 预测为例, 著名的 ADMET 商业软件中的 $\mathrm{p} K_{\mathrm{a}}$ 预测模块就是通过神经网络 集成实现的 ${ }^{[68]} .2019$ 年, Grzybowski 等 ${ }^{[69]}$ 通过图卷积神 经网络(Graph convolutional neural network, GCNN)实现 了 $\mathrm{C}-\mathrm{H}$ 酸 $\mathrm{p} K_{\mathrm{a}}$ 在毫秒级时间的准确预测, 对大约 13000 个去质子反应的预测正确率大于 $90 \%$, 平均绝对误差 $\mathrm{MAE} \approx 2.1$. 最近, 罗三中和张龙等 ${ }^{[70]}$ 基于机器学习方 法建立了 $\mathrm{p} K_{\mathrm{a}}$ 的全局预测模型(图 4). 他们从 $i \operatorname{BonD}^{[71]}$ 数据库中收集了 39 种溶剂的 $\mathrm{p} K_{\mathrm{a}}$ 实验数据, 清洗和整理 后得到了包含 15338 种化合物的数据集. 在描述符方面, 他们开发了将分子指纹和物理有机参数相结合的 SPOC (Structure and physical organic chemistry)描述符. 在建模 方面, 他们选择 5 重交叉验证方法对常用机器学习方法 进行了篮选, 发现运用神经网络或XGBoost算法训练的 全局模型具有最佳预测表现, MAE 仅为 0.87 个 $\mathrm{p} K$ 单位. 并可以实现多溶剂体系 $\mathrm{p} K_{\mathrm{a}}$ 的快速精准预测, 该研究还 表明, 全局模型的预测结果优于所有的单一溶剂模型, 对不同溶剂中 $\mathrm{p} K_{\mathrm{a}}$ 预测值的相关度分析也验证了迁移学 习的特征. 此外, 对样本外药物分子, 二甲基亚砜 (DMSO)中氢键催化剂以及乙腈中氨基催化剂 $\mathrm{p} K_{\mathrm{a}}$ 预测 进一步验证了该模型的稳健性.

最近, 神经网络算法也被应用于化学键均裂能 (BDE) 预测中. 江俊等 ${ }^{[73]}$ 以原子种类和电荷分布为描述 符, 采用 3 个隐藏层的神经网络对 8000 个有机化合物的

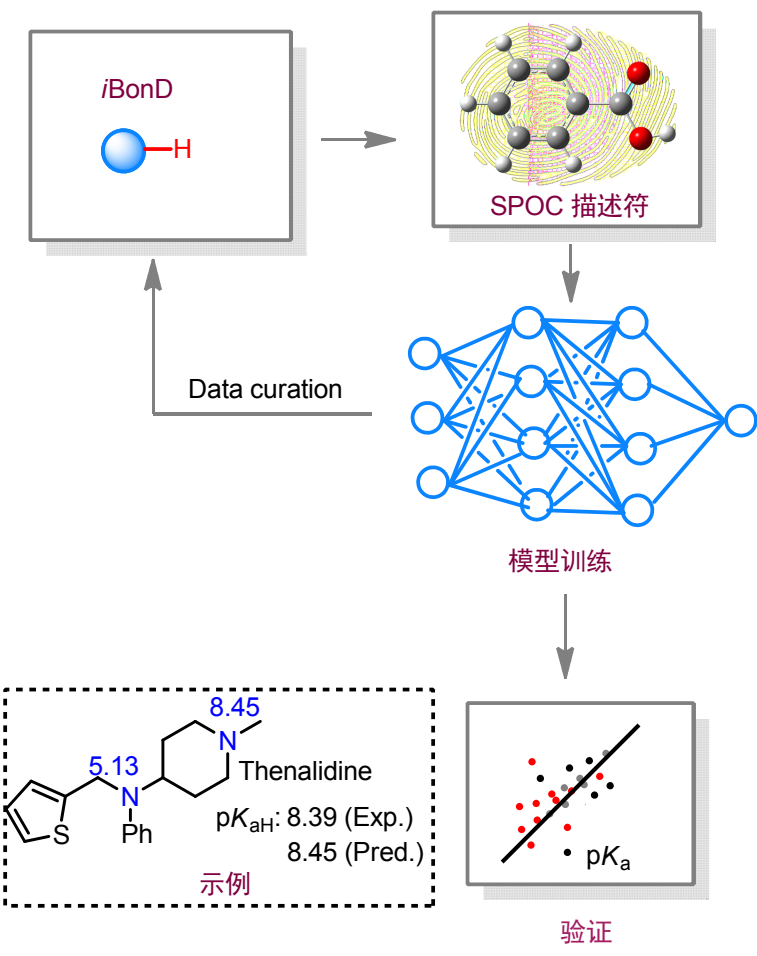

图 4 建立 $i \operatorname{BonD} \mathrm{p} K_{\mathrm{a}}$ 模型的工作流程 ${ }^{[67]}$

Figure 4 Workflow for the construction of $i \mathrm{BonD} \mathrm{p} K_{\mathrm{a}}$ model

量化计算 BDE 数据进行了建模和预测, 其预测的平均 误差为 $4.05 \mathrm{~kJ} / \mathrm{mol}$, 相关系数 $R^{2}$ 可达 0.97 , 与 DFT 计算 结果相当. 同样, 针对其他量化计算所得的化合物性质 如频率、零点能、最高已占分子轨道(HOMO)或最低空 分子轨道(LUMO)能量等也可以通过机器学习预测快速 获得. 2017 年, 谷歌的研究人员 ${ }^{[74]}$ 从几种流行的处理图 结构数据的神经网络模型中抽象出共性, 提出了一种应 用于图结构的监督学习框架，该框架称为消息传递神经 网络(Message passing neural networks, MPNNs). 通过对 QM9 数据库中的 DFT 计算数据进行建模, 成功实现了 包括原子化能、红外振动频率、零点振动能和 HOMOLUMO 能量等有机分子量子化学性质的预测.

\section{2 分子从头设计}

分子从头设计(De novo design)利用算法虚拟设计 和评估一系列符合特定性质的分子，可用于药物、材料 等功能分子的发现 ${ }^{[10,75]}$. Gómez-Bombarelli 等 ${ }^{[10 b]}$ 发展了 使用变分自编码器(Variational autoencoder, VAE)生成分 子结构的方法. 他们从 ZINC 数据库获取分子并以 SMILES 格式输入, 编码器将分子的这种离散表示转换 为隐含空间的连续向量, 解码器再将这些连续向量还原 成分子 SMILES(图 5). 此方法的隐含空间中的分子表示 是连续的，因此可以通过随机解码、扰乱或插入等方法 产生新的分子，并通过一些优化算法产生特定性质的分 子 ${ }^{[76] .} 2017$ 年, Kadurin 等 ${ }^{[77]}$ 使用 VAE 结合生成对抗网 
络 ${ }^{[78]}$, 生成了具有特定抗癌活性的分子. 2019 年, Zhavoronkov 等 ${ }^{[79]}$ 基于 VAE 实现了盘状结构域受体 1 (Discoidin domain receptor 1, DDR1) 有效抑制剂的快速 设计.

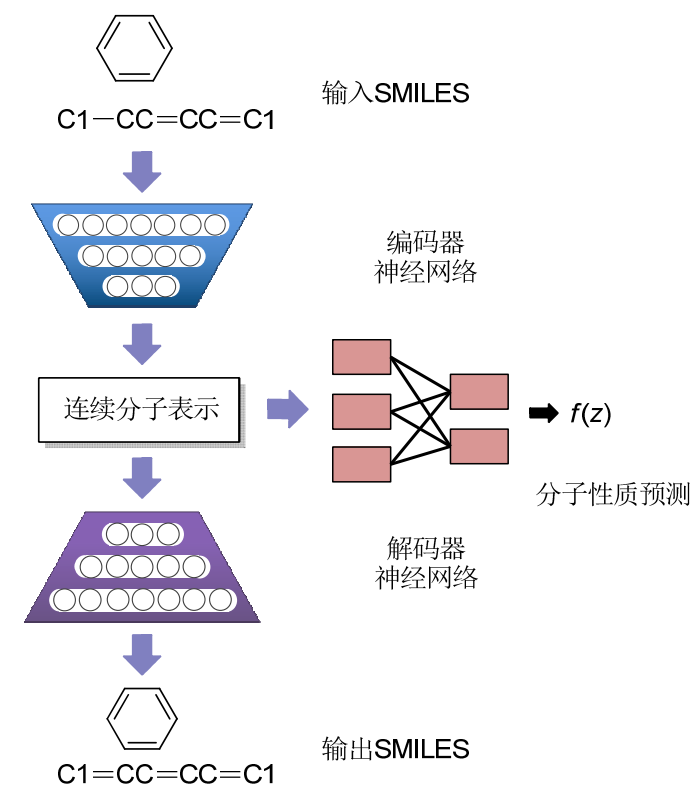

图 5 用于分子从头设计的自编码器

Figure 5 A diagram of the autoencoder used for molecular design

近年来, 使用 RNN 模型进行分子从头设计受到了 越来越多的关注. 2017 年, Segler 等 ${ }^{[10 \mathrm{c}, 80]}$ 报道了使用 RNN 生成新型分子结构. 通过使用大量 SMILES 训练 RNN网络并学习 SMILES 的概率分布, 该网络可以高效 生成训练集之外的各种分子结构(图 6). Segler 等尝试预 先在一个通用集上训练神经网络模型, 随后通过迁移学 习将预训练的模型应用于特定数据集, 以提高小数据集 的预测性能. 通过这一策略, 该模型能生成 $14 \%$ 从未出 现过的抗金色葡萄球菌和 $28 \%$ 新型的抗疮疾的活性分 子.

2016 年, Jaques 等 ${ }^{[81]}$ 使用一种强化学习算法 Deep Q-learning 结合预先训练的 RNN 模型, 生成了具有特定
分子性质(比如 $c \log P^{[82]}$ 和 $\mathrm{QED}$ drug-likeness ${ }^{[83]}$ )的新型 分子. 然而，该方法依赖于包含手写规则的奖励函数, 也可能生成没有真实意义的简单分子结构. 为解决此问 题, Olivecrona 等 ${ }^{[84]}$ 提出了一种基于策略的强化学习方 法，通过预训练 RNN，可以生成目标结构的类似物和对 生物靶标有预期活性的分子. 此模型被用于训练生成药 物塞来昔布的类似物，在另一项训练任务中，该模型能 够以 $95 \%$ 以上的准确率生成对多巴胺 II 型受体有效的 分子.

从上述工作中可以看出, 机器学习算法, 特别是深 度神经网络算法, 可以根据实际需要来定义分子特性并 生成具有较高多样性和特异性的分子. 然而，对抗神经 网络和增强学习方法容易陷入局域分子空间，可能导致 生成的分子仅仅是某类分子骨架的简单变化 ${ }^{[85]}$, 最近 越来越多的工作开始关注并尝试解决这些问题 ${ }^{[86]}$.

\section{3 化学反应预测}

关于反应预测和逆合成分析的研究起步较早, 自 20 世纪 60 年代开始就陆续出现了若干计算机辅助合成 设计系统，例如 LHASA $^{[87]}$ 和 SYNGEN ${ }^{[88]}$ 等. 然而，传 统的方法使用大量专家制定的规则来判断某条路径的 可行性，并没有得到完全令人满意的结果 ${ }^{[89]}$. 最近, 基 于机器学习方法的反应预测研究进展迅速 ${ }^{[90]}$. 下面将 简要介绍机器学习算法在前向反应预测、反应活性与选 择性预测，以及反应条件预测方面的应用进展.

\subsection{1 前向反应预测}

2011 年, Baldi 课题组 ${ }^{[91]}$ 使用自定义的分子轨道概 念(Unfilled and filled molecular orbitals)和物理化学描述 符作为输入, 通过对反应数据进行训练, 最终能以 89.05\%的精度预测极性反应. 如果综合考虑前 4 种可 能，预测精度能进一步提升到 $99.86 \%$ (图 7). 该策略考 虑了具体的反应条件，因而能得出更加真实可信的结 果. 同时，该策略在一定程度上从机理层面阐释了化学 反应中电子转移的基元过程, 并能识别和预测多步反应 过程. 随后, Baldi 课题组 ${ }^{[92]}$ 将这一方法进一步推广到自
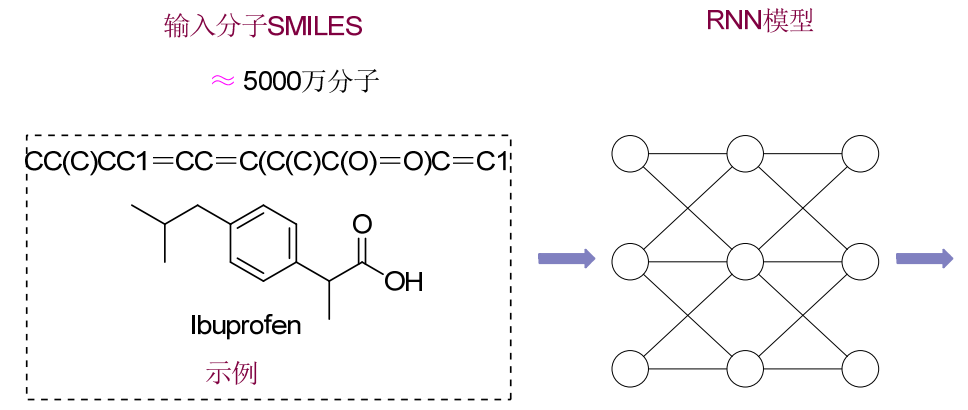

输出新分子结构

图 6 用于分子从头设计的 RNN 模型

Figure 6 RNN model for molecular de novo design 


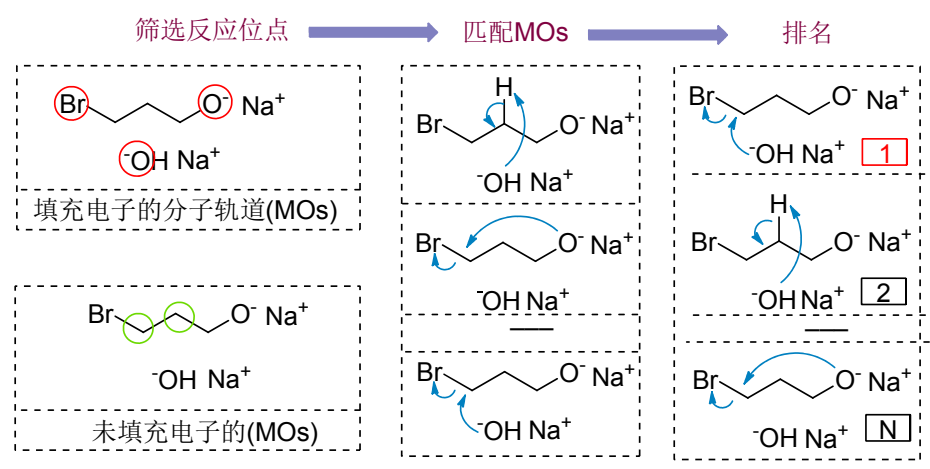

图 7 Baldi 等的化学反应预测框架

Figure 7 Chemical reaction prediction framework by Baldi

由基反应和周环反应中.

利用图卷积神经网络预测反应物到产物的原子和 化学键变化也是一种反应预测的机器学习策略. 2019 年, Jensen 等 ${ }^{[58 b]}$ 使用分子图表示反应物分子. 分子图的 节点和边分别描述原子和化学键, 通过图卷积神经网络 计算了每个原子对之间化学键变化的可能性, 可能性大 的候选产物被组合列举出来并通过另一个图卷积网络 重新预测出主要产物的概率分布. 他们对来自专利文献 中数十万个反应进行训练, 最终准确预测了 $85 \%$ 以上的 主要产物(图 8).

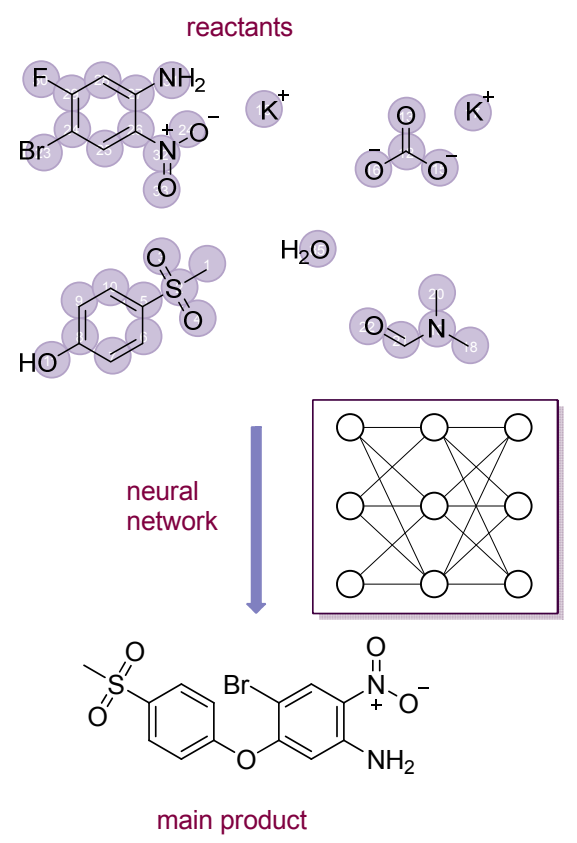

图 8 Jensen 等的化学反应预测框架

Figure 8 Chemical reaction prediction framework by Jensen

除了上述的方法, 预测化合物 SMILES 的序列到序 列模型(Sequence-to-sequence model)也被应用于反应预 测. 基于序列的技术的关键思想是使用反应物、试剂和 产物的文本表示(通常是 SMILES), 并将反应预测视为 从一种语言(反应物-试剂)到另一种语言(产物)的机器
翻译问题. 2019 年, Schwaller 等 ${ }^{[93]}$ 使用多头注意力 (Multihead attention)的 Transformer 模型, 不需要手动创 建规则, 只是通过推断数据集中的反应物、试剂、产物 中化学结构之间的相关性来进行预测. 他们将 SMILES 作为输入，模型在一个通用的基准数据集上排名第一的 预测结果精度达到了 $90.4 \%$.

\subsection{2 反应活性与选择性预测}

给定反应物和反应条件, 机器学习策略也能实现反 应活性与选择性的预测. 早期工作大多使用 HammetTaft 等自由能参数来描述反应特征 ${ }^{[94]}$, 成功实现了对 $\alpha, \beta$-不饱和化合物迈克尔加成反应活性的预测 ${ }^{[95]}$. 后续 也有使用分子拓扑描述符 ${ }^{[96]}$ 和量子化学描述符 ${ }^{[97]}$, 或 者综合两种方法 ${ }^{[8]}$ 研究反应物、反应条件与反应活性之 间的关系的报道; 基于分子描述符和位阻参数的多元线 性回归模型可以预测反应活性 ${ }^{[99]}$; 也有报道利用反应 位点电子转移模式预测化学反应速率 ${ }^{[100]}$. 采用氢键、位 阻以及理论计算得到的振动频率、NPA 电荷等作为描述 符非常适用于反应选择性预测，这方面工作已有相关总 结 ${ }^{[101]}$, 在此不再赘述. 由于反应条件对反应活性的影 响是一个复杂的问题, 不同的反应在不同条件下的反应 机理可能完全不同，因此很难找到一类通用的方法描述 该过程 ${ }^{[102]}$. 机器学习方法能够从实验人员无法理解的 大量数据集里发现反应模式, 这些构效关系的发现有助 于发现高效催化剂, 从而快速优化催化反应 ${ }^{[59]}$.

近年来，传统物理有机线性自由能关系被成功应用 于手性催化反应定量构效关系(Quantitative structurereactivity relationships, QSRR)模型, 实现了对一系列手 性金属配合物催化反应活性和对映选择性的精准预测, 指导了催化剂的结构优化 ${ }^{[103]}$. 但这些研究都只针对单 一催化体系, 模型的普适性和拓展能力有限. 2019 年, Denmark 等 ${ }^{[42]}$ 运用支持向量机和深度前馈神经网络预 测了手性磷酸催化的硫醇与酰亚胺的加成反应的选择 性. 他们选择了 5 种硫醇底物、 5 种酰亚胺底物和 43 种 磷酸催化剂生成的 1075 个实验数据作为训练和验证集. 
使用基于 3D-格点的 ASO 描述符(Average steric occupancy, 平均空间占有率)来描述分子的空间特征, 并加 入取代基的静电势能面最大值(Electrostatic potential energy maximum, ESPMAX)作为电子描述符. 在训练集 选取方面, 他们通过 Kennard- Stone 算法抽样选出具有 代表性的 23 种催化剂子集作为训练集 UTS (Universal training set), 该方法可以保证训练集特征在整个催化空 间的均匀分布. 最终模型以较高的精度预测了产物的 $e e$ 值, MAE 在 $0.995 \mathrm{~kJ} / \mathrm{mol}$ 以内, 并且成功预测了一种最 优的大位阻磷酸催化剂(图 9), 显示了该策略在预测新 颖催化剂方面的潜力.

尽管随机森林算法已有 20 多年的发展历史, 但其 在当前的深度神经网络时代仍然具有竞争力. 近期, 随 机森林算法在催化反应预测方面表现不俗. 2018 年, Doyle 等 ${ }^{[104]}$ 报道了随机森林模型对 Buchwald-Hartwig
胺化反应产率的预测. 通过高通量实验得到了 23 种异 噁唑添加剂、 15 种芳基和杂芳基卤化物、4 种钯催化剂 和 3 种碱所组成的 4608 个反应数据(图 10), 并提取了 120 种原子、分子和振动描述符作为输入. 该模型以均 方根误差 $(\mathrm{RMSE})=11.3 \%, R^{2}=0.83$ 的精度预测了产率.

Sunoj 等 ${ }^{[105]}$ 同样利用随机森林算法实现了不对称 催化氢化反应对映选择性的预测. 模型数据集包含了 58 种轴手性联萗配体、190 种烯烃和亚胺共 368 个反应 数据, 9 种分子参数 (如键长、键角、二面角) 和 22 种代 表整体分子特性的描述符(如 HOMO 和 LUMO 能量、 偶极矩) 作为模型的参数. 与实验值相比, 随机森林算 法能准确预测对映选择性, 其 RMSE 在 $(8.4 \pm 1.8) \%$ 范围 内.

洪金等 ${ }^{[106]}$ 对杂环自由基 $\mathrm{C}-\mathrm{H}$ 官能团化的区域选 择性进行了预测. 模型的训练中使用了分子特征库，包

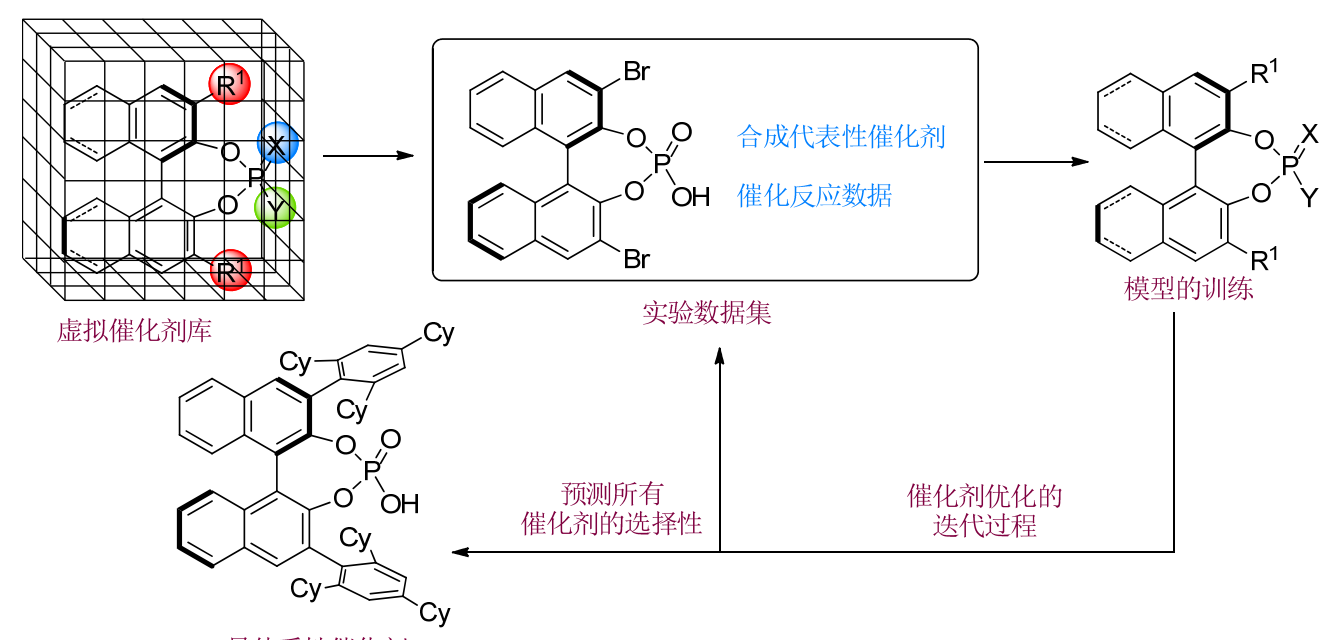

图 9 预测高选择性催化剂的工作流程

Figure 9 Workflow of the prediction of higher-selectivity catalysts
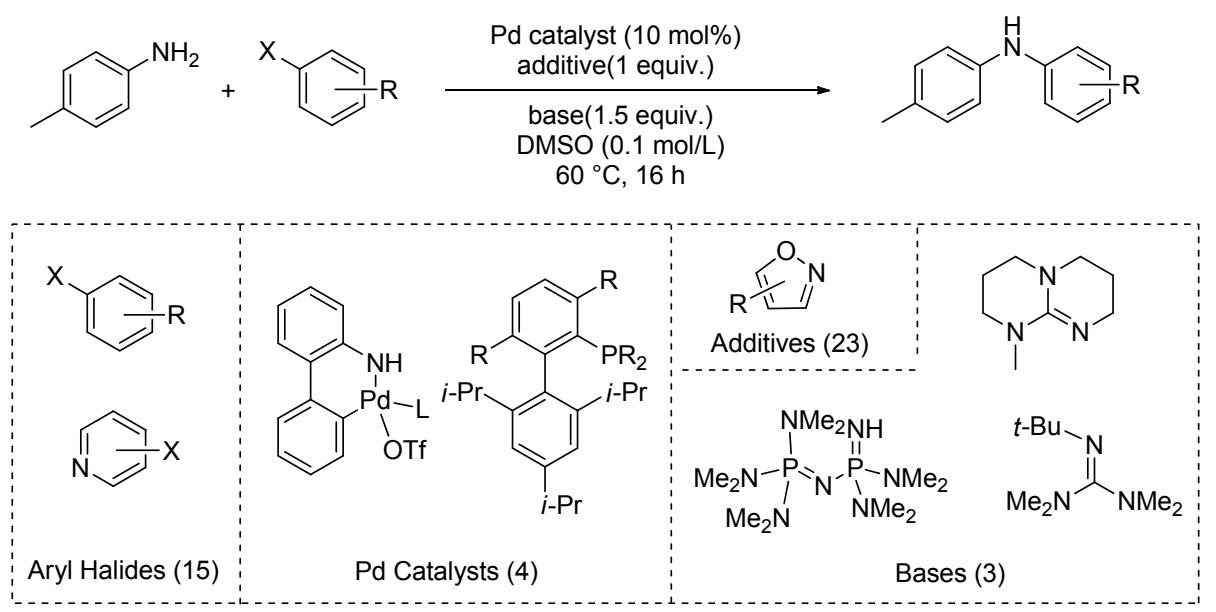

图 10 钯催化的 Buchwald-Hartwig 反应产率预测

Figure 10 Prediction of yield in palladium catalyzed Buchwald-Hartwig reaction 
含 $\mathrm{C}-\mathrm{C}$ 键形成过程的局部原子特性、自由基的整体分 子特性以及物理有机化学描述符. 通过物理有机特性与 随机森林算法相结合, 建立了一个将 DFT 计算的孤立 反应物性质与过渡态能垒联系起来的回归模型, 样本外 测试集的预测结果显示位点预测精度为 $94.2 \%$, 选择性 预测精度为 $89.9 \%$.

不同于上述采用物理化学意义明确的参数或描述 符的工作, Glorius 等 ${ }^{[107]}$ 最近发展了一种基于多指纹特 征(Multiple fingerprint features, MFFs)的分子表示方法. 同样采用随机森林算法, 使用上文 Denmark 数据集实现 了对映选择性的预测, MAE 在 $1.179 \mathrm{~kJ} / \mathrm{mol}$ 以内. 该方 法还能够预测反应产率和反应性. 采用分子指纹方法构 建的模型计算成本低、具有一定的普适性, 但在模型的 可解释性方面可能需要进一步分析.

\subsection{3 反应条件的预测与优化}

传统的初始反应条件选择基本是经验性的或直接 参考文献信息. 然而, 化学信息的指数增长使人工分析 和总结变得极其困难, 需要专门的方法和工具来有效地 从原始数据中提取这些知识 ${ }^{[108]}$. 因此, 发展一种高效、 快速的反应条件管选策略具有重要的科学意义. 由于化 合物的反应性很大程度上是由反应条件决定的，因此反 应条件的理论评估尤为重要. 到目前为止, 已经报道了 几种不同的反应条件建模方法. 2015 年, Marcou 等 ${ }^{[109]}$ 使用反应压缩图(CGR)衍生片段描述符，构建了支持向 量机、随机森林和朴素贝叶斯分类模型来预测共轭加成 反应的溶剂和催化剂的最佳组合. 随后, Varnek 等 ${ }^{[108]}$ 基 于相似的反应在相似的条件下进行的原理, 运用 CGR 表示化学反应，预测的保护基团脱保护反应最佳实验条 件具有较高的准确性(约 $90 \%$ ). 为准确预测适用于大型 反应体系的完整反应条件, 2018 年, Jensen 等 ${ }^{[110]}$ 发展了 一种分层设计的神经网络模型来预测化学环境(催化 剂、溶剂、试剂)和反应温度(图 11). 该模型对约 1000 万个来自 Reaxys 的反应进行了训练, 在训练集以外的 100 万个反应中进行了测试，以 $69.6 \%$ 的准确率预测了 排名前十的反应试剂，以 $60 \% \sim 70 \%$ 的准确率预测了反 应温度 $\left( \pm 20{ }^{\circ} \mathrm{C}\right)$.

未经优化的化学反应在反应时间、试剂方面经常面 临低效和成本的问题. 优化反应的一种常用方法是一次 改变一个实验条件, 同时固定所有其他条件, 该方法常 会错过最佳条件; 另一种方法是通过组合化学篮选反应 条件的所有组合, 虽然这种方法有更大可能找到全局最 优条件, 但是费时费力. 因此, 通过机器学习方法构建 有效的反应条件优化体系, 对学术研究和工业生产都具 有重要意义. 溶剂选择作为一个独立的问题在早期得到 了广泛的研究 ${ }^{[110]} .2013$ 年, Struebing 等 ${ }^{[111]}$ 将基于 DFT

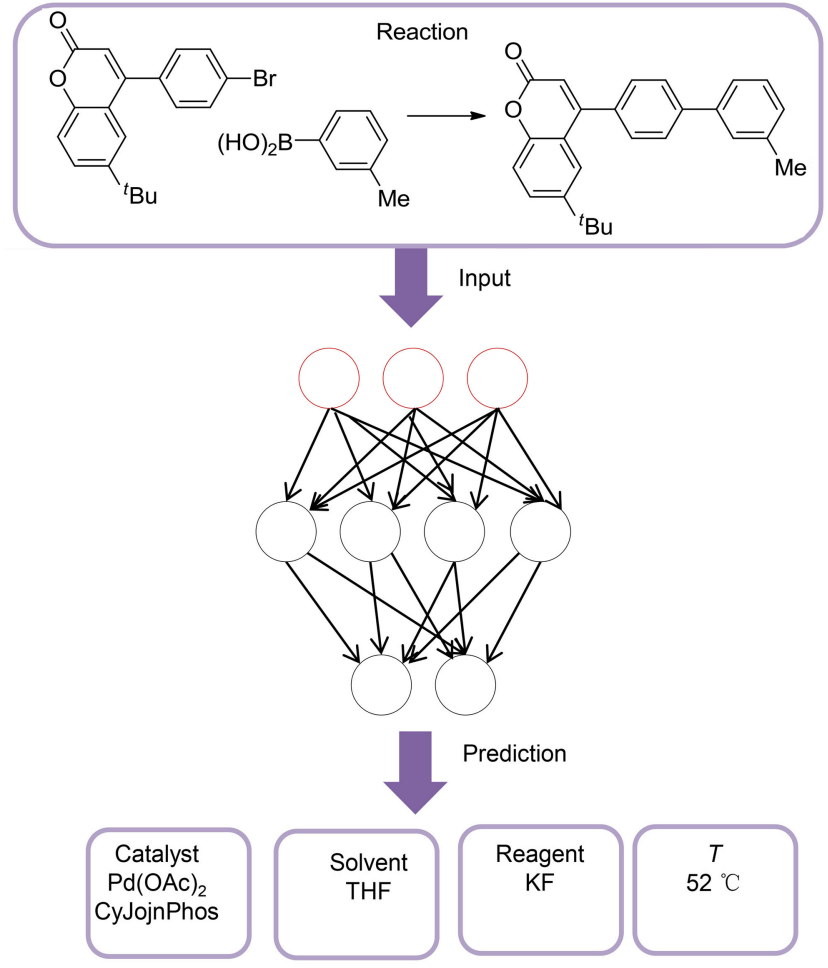

图 11 分层设计的神经网络模型预测反应条件 Figure 11 Neural network model of layered design for predicting reaction conditions

的速率常数计算与线性回归模型相结合, 对反应溶剂进 行优化，在 1341 种溶剂的搜索空间内测试了 9 种溶剂, 将 Menschutkin 反应的速率常数提高了 $40 \% .2018$ 年, Aspuru-Guzik 等 ${ }^{[12]}$ 开发了名为 Phoenics 的主动学习算 法，其将贝叶斯优化与贝叶斯核密度估计 (Bayesian kernel density estimation)相结合, 实验过程可以按照 Phoenics 所提出的反应条件执行, 再将实验结果重新输 入程序. 通过此种反馈机制, Phoenics 可以提出一系列 实验条件集并最终确定最佳条件集.

\section{4 逆合成分析}

计算机辅助合成设计的目的是确定一系列可行的 反应步骤，从而由可获取的原料合成目标产物. Corey 等 ${ }^{\left[{ }^{[0]}\right.}$ 开发的逆合成方法考虑了逆向策略, 即从产物出 发，搜索可能的前体。一些综述介绍了各种逆合成方法 和工具的历史 ${ }^{[89 a, 113]}$, 在此不再详细介绍. 本文只对当 前常用的逆合成策略: 基于模板(Template-based)和无模 板(Template-free)两种逆合成策略的发展做一简介.

基于模板的逆合成方法将反应规则与目标分子匹 配以产生一个或多个候选前体 ${ }^{[114]}$. 模板可以由专家整 理或者从反应数据库中自动提取 ${ }^{[115]}$. CHEMATICA 软 件 ${ }^{[86]}$ 整合了超过一万条有机化学家手动编辑的经验反 应规则，该反应规则与启发式策略一起使用，以确定合 
成路径. 从数据库中自动提取反应规则是当前更主流的 方式. 2019 年, Law 等 ${ }^{[116]}$ 按照 “处理反应数据-建立原 子/原子映射一识别反应中心及其外围环境” 的流程建立 了 ARChem 合成路线设计系统. 2017 年, Coley 等 ${ }^{[117]}$ 表 明, 模板可以通过目标分子与数据库中反应产物之间的 分子相似性(Molecular similarity)进行排序.

2017 年, Waller 等 ${ }^{[11]}$ 运用深度神经网络模型对收集 的 350 万个反应数据进行训练. 该模型可以自动提取反 应规则，在近 100 万个反应的验证集中预测的前十个目 标反应的命中率达到了 $97 \%$. 随后, Waller 课题组尝试 使用蒙特卡洛树搜索和深度神经网络策略, 搜索 40 种 药物类似分子的合成路线. 与之前发展的方法相比, 该 策略能以更高的精度推测可行的合成路线(与基准预测 的 $87.5 \%$ 的准确率相比, 该策略的准确率达 $95 \%$ ). 随后, Waller 课题组 ${ }^{[11]}$ 结合策略网络和蒙特卡洛树搜索对 1200 万反应数据进行训练(图 12), 结果表明该策略显著 加速了逆合成路线预测的速度和准确率. 盲测结果表明 受试者很难分辨出模型给出的逆合成路线与人类专家
设计的逆合成路线. 当使用 USPTO 的 5 万组反应数据 作为测试集时, 模型也能取得较高的预测精度.

无模板的逆合成方法中, 化学结构的所有转变是在 没有任何反应规则的情况下进行的, 其中基于深度学习 的策略越来越流行. 2017 年，Liu 等 ${ }^{[118]}$ 建立了将反应产 物 SMILES 转换为反应物 SMILES 的序列到序列模型, 该模型以美国专利文献中的 50000 个实验反应实例为训 练样本, 与基于规则的专家系统基准模型的性能相当. 最近, 来鲁华和裴剑锋等 ${ }^{[119]}$ 提出了一种无模板方法, 使用 Transformer 框架构建了单步逆合成分析模型，并 结合蒙特卡洛树搜索(MCTS)和启发式打分函数建立了 逆合成路线规划系统 AutoSynRoute(图 13), 该系统成功 实现了 4 种分子的逆合成路线设计.

当前, 前向反应预测主要用于预测反应产物、潜在 的副产物和杂质，而将其与逆合成分析相结合用于反应 路径的工作较少. 最近, Schwaller 等 ${ }^{[120]}$ 使用前向预测模 型 ${ }^{[121]}$ 和反应分类模型 ${ }^{[122]}$ 来评估逆合成预测的质量, 提 升了单步逆合成模型的性能.

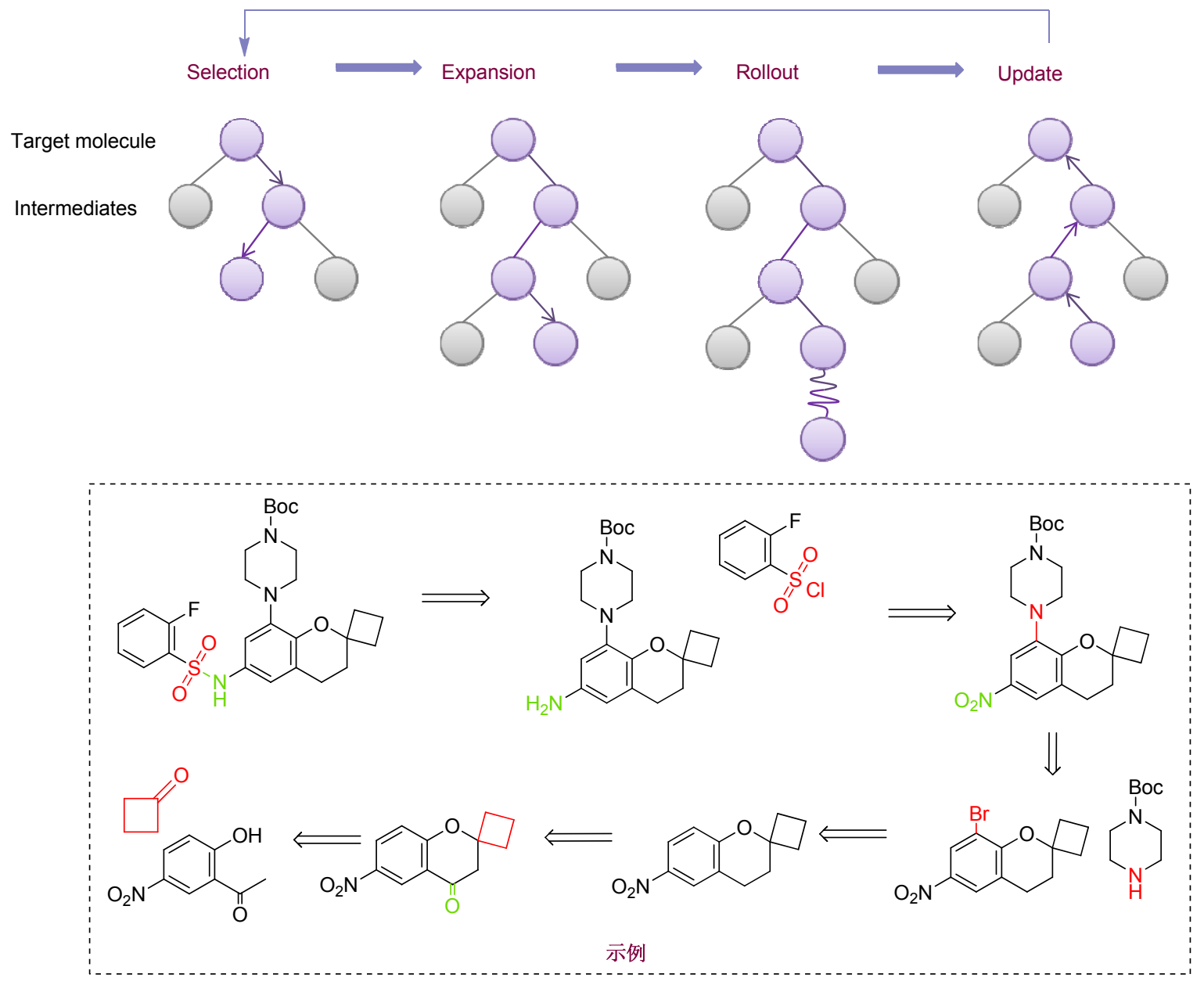

图 12 用于逆合成分析的 MCTS 方法

Figure 12 MCTS method for retrosynthetic analysis 


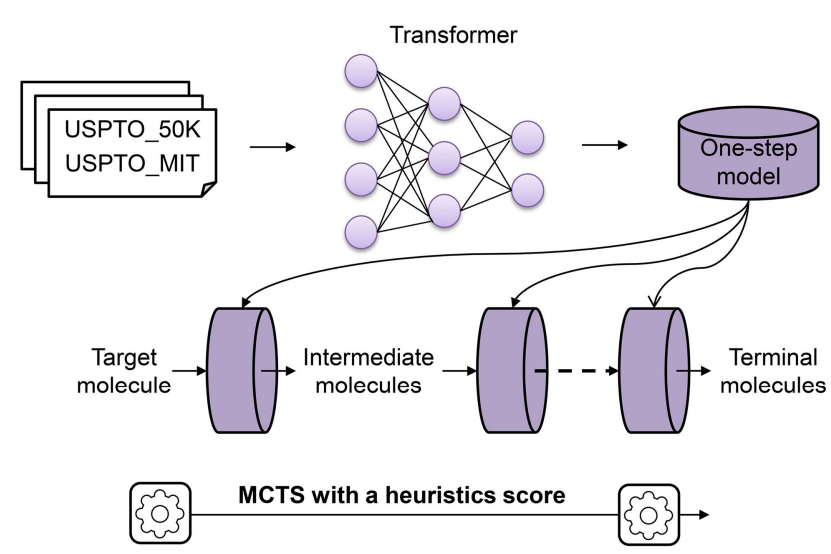

图 13 AutoSynRoute 的工作流程

Figure 13 Workflow of AutoSynRoute

\section{5 智能合成机器}

智能合成机器是合成化学研究发展的长期目标, 旨 在从根本上改善合成化学的效率和成本, 能够辅助化学 家进行重复性、高成本或危险性的实验. 随着自动化化

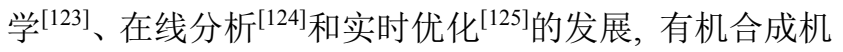
器已经初露锋芒, 通过建立机器学习算法控制的反应系 统, 可以开发出能够自主探索化学反应性的智能合成装 置 ${ }^{[12]}$.

2018 年, Perera 等 ${ }^{[126]}$ 的工作表明流动装置可以在药 物发现过程的早期加速反应优化, 并提供了可靠的数据 帮助其他实验室建立机器学习算法. 随后, Felpin 等 ${ }^{[127]}$ 提出了一种将监测技术与反馈算法相结合的模块化自 动流动反应器. 该自动化反应装置通过在线高效液相色 谱(HPLC)和在线 NMR 检测产物, 并根据结果自动优化 条件, 通过尝试 66 种实验组合, 最终通过四步反应, 以 67\%的产率合成了天然产物 Carpanone.

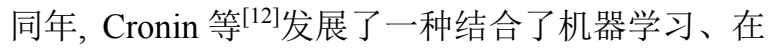
线核磁、红外、质谱的自动化反应篮选装置. 他们的工 作表明, 由机器学习算法控制的自动反应处理系统可能 比人工处理反应快一个数量级. 这种方法能够以结构化 的方式寻找失败或无反应性的实验信息. 机器学习算法 能够对来自辉瑞数据集的 1000 个钯催化的 SuzukiMiyaura 反应组合的反应性进行预测, 在考虑了略多于 $10 \%$ 的数据集的结果后, 精度超过 $80 \%$. 为了实现反应 装置的模块化和自动化, 他们之后设计了一套基于化学 编程语言的自动化合成系统 Chemputer, 该系统通过标 准的化学语言控制和协调各反应模块, 最终实现化合物 的多步合成 ${ }^{[128]}$. 这一策略是自动化合成与化学反应模 块化和标准化的一个很好范例.

2019 年, Jamison 和 Jensen 等 ${ }^{\left[{ }^{[29]}\right.}$ 开发了一种基于人 工智能的自动化合成机器人平台(图 14). 他们首先开发
了合成规划软件 ASKCOS，该软件采用基于模板的逆 合成方法, 从 USPTO 和 Reaxys 数据库收集的反应数据 中提取转换规则, 用于训练前馈神经网络模型, 最终的 合成路线选择包括逆向综合规划、反应条件推荐和路径 评估，部分路线包含化学配方文件(Chemical recipe files, CRFs), 需要用户提供额外的信息来确定停留时 间、化学计量和浓度. 之后, 他们设计了一个模块化的 连续流动平台来执行这些文件，根据 CRF 中确定的合 成路线, 机械臂将模块化过程单元(反应器和分离器)组 装成连续流动路径, 实现了 15 种药物或类药物分子的 自动化合成. 该平台将 CASP 与机器流动化学平台相结 合，加速了小分子的自动合成，但合成路线仍需要人工

(a)

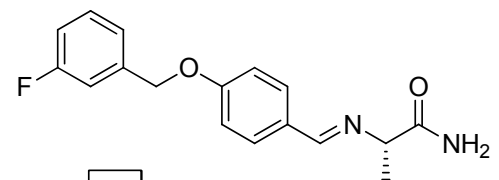

A 目标分子
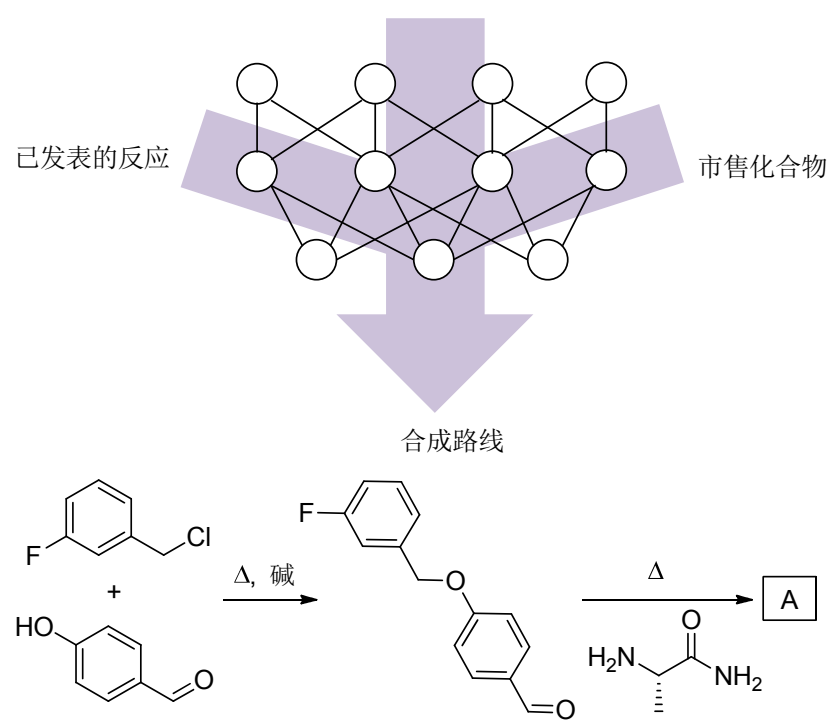

(b)

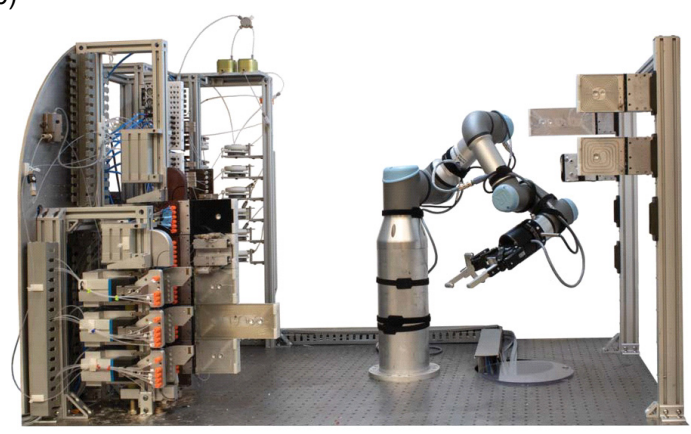

图 14 Jamison 和 Jensen 等开发的自动化合成机器人平台 Figure 14 Automated synthetic robot platform developed by Jamison and Jensen et al.

(a) Workflow of synthetic planning software. (b) mobile chemical robot platform ${ }^{[129]}$. Copyright 2019 American Association for the Advancement of Science 
输入. 最近, Gilmore 等 ${ }^{[130]}$ 报道了一种有机分子的自动 化径向合成(Radial synthesis)仪器, 该仪器由四部分组 成: 溶剂和试剂输送系统 (Reagent delivery system, RDS)、中央交换站(Central switching station, CSS)、备用 模块 (Standby module, SM) 以及收集容器 (Collection vessel, CV), 一系列连续流动模块被径向布置在 CSS 周 围. 这种全自动仪器能够进行线性和循环合成, 不同过 程之间无需重新手动配置.

\section{3 挑战与机遇}

\section{1 数据与模型的开放和共享}

当前, 虽然有一些免费开放的化学信息学数据 库 $^{[131]}$, 但这类数据库数量仍然较少, 数据也不够完善. 机器学习在化学领域的发展仍然受数据集规模和质量 的限制. 同时也需要建立更多标准化学数据库, 方便不 同机器学习方法的验证和比较. 在图像识别领域, ImageNet 数据库 ${ }^{[132]}$ 的建立推动了该领域快速发展. 受 ImageNet 和 $\mathrm{WordNet}^{[133]}$ 启发, $\mathrm{Wu}$ 等 ${ }^{[134]}$ 建立了 MoleculeNet 数据集, MoleculeNet 基于 DeepChem 开源 软件包并提供相关接口, 收集汇总了大量包含量子化 学、物理化学、生物物理和生理学信息的数据集, 并发 展了处理分子表示和机器学习算法的全套流程, 该数据 集将有助于新算法的开发与横向比较.

数据的共享也是当前面临的一个挑战. 研究工作的 代码和数据集应尽可能公开, 这不仅能方便基准测试, 更能够促进机器学习算法的开发. 为推动数据的开放与 共享, 开发化学语言标准是十分必要的. 化学标记语言 (Chemical markup language, CML)是一种代表性的通用 化学语言标记格式, 可以表示分子、反应、光谱、分析 数据和实验操作流程等信息, 目前在化学专利数据库的 标注和数据挖掘领域已有广泛应用 ${ }^{[19]}$.

另一个挑战是如何通过文献报告机器学习工作是 如何完成的, 以便其他人可以重复 ${ }^{[135]}$. 机器学习模型 参数众多, 程序结构复杂, 数据庞大, 很难通过简单的 流程图或文本描述所有细节, 文献和补充材料无法实现 有效的参数和代码共享. 因此, 有必要开发和采用新的 数据和代码共享方法来确保机器学习方法在此领域的 可用性.

如果 Reaxys 和 SciFinder 等数据库能够开放接口给 化学工作者们使用, 同时化学工作者共享最新实验数 据, 结合数据的标准化, 将极大推动有机化学领域机器 学习的发展. 未来, 化学界应该努力打破不同研究机 构、高校和组织之间的数据壁垒, 以更加开放的姿态共 同推动化学领域的理性化进程.

\section{2 高质量实验数据的收集整理}

Norquist等 ${ }^{[136]}$ 指出, 机器学习程序可以从失败的实 验数据中学习并成功用于反应预测, 因而失败的反应数 据对于机器学习理解反应非常重要. 然而, 记录文献报 道的数据库通常只包括较为成功的实验数据. 大多数反 应预测都是基于成功的反应(例如 USPTO 和 Reaxys 数 据集)进行训练的, 因而模型很难从这些数据集中获取 更多有效信息. 此外，由于识别所有化学物质的时间和 成本都很高, 因此反应混合物中副产物的全部表征并不 经常公开，这限制了预测反应性模型的构建 ${ }^{[137]}$.

电子实验记录本作为一种有效的实验记录手段, 记 录了包括大量成功和失败的反应等的实验室第一手数 据. 近年来, 越来越多的电子实验记录本被开发出来, 但是由于实验人员的传统思维和对新技术的谨慎, 电子 实验记录本除了在有规定性要求的公司中使用以外, 其 普及率仍然较低. 电子实验记录本的普及使用将有助于 改善数据集中失败数据贵乏的问题, 从而推动机器学习 整体预测能力的提高.

除此之外, 实验数据的质量与可重复性也是一个挑 战. 近期发展的反应自动化是一种获取可重复性和标准 化数据的有效方法 ${ }^{[12,138]}$, 通过模块化实验装置、标准化 操作和标准化学语言, 该策略排除了个体和环境差异, 实验结果的可靠性和重复性更高. 此外, 通过将实验和 分析数据整合到一个统一的数据平台, 研究者可以方便 获取更多高质量和多维度的实验数据, 这将大大促进使 用机器学习方法进行数据挖掘和反应预测的发展 ${ }^{[139]}$.

\section{3 描述符和机器学习算法的发展}

如何有效地表示分子结构和化学反应也是机器学 习面临的挑战之一. 大多数应用于化学反应或性质的机 器学习方法使用分子或原子描述符来建立模型, 模型的 成功与否取决于这些描述符的有效性和相关性. 利用神 经网络为反应中的分子创建指纹等进展推动了反应预 测的改进 ${ }^{[140]}$. 提高机器学习方法的性能需要开发新的 化学描述符.

机器学习算法面临的一个挑战是如何从较小的数 据集中获得更多知识. 机器学习方法通常需要大量的数 据才能有效地学习. 虽然这在图像识别等领域很少是一 个问题, 但在化学领域通常仅限于数百或数千个高质量 的数据. 有限数据集问题的一个有希望的解决方案是元 学习, 即在问题内部和问题之间学习 ${ }^{[141]}$. 诸如神经图 灵机 ${ }^{[142]}$ 和模仿学习 ${ }^{[143]}$ 等新的发展使这一过程得以实 现. 最近有报道称，贝叶斯程序学习(Bayesian program learning, BPL)能够在数据有限的情况下对 one-shot 分类 任务表现出接近人类水平的性能 ${ }^{[144]}$.

匹配网络(Matching networks) ${ }^{[145]}$ 可以使用辅助数 
据预先训练, 并引入记忆机制和注意力机制, 从而提高 小数据集下的训练表现. 类似的方法通常用于药物化学 领域, 因为新型靶标的信息和数据较少, 使用传统的机 器学习算法很难通过少量数据训练出稳定可靠的模型. 2017 年, Altae-Tran 等 ${ }^{[146]}$ 使用长短期记忆 (long short-term memory, LSTM)方法实现了小数据集下的模 型训练. 最近, 记忆增强神经网络(Memory augmented neural networks)通过在传统的神经网络模型里增加记忆 模块, 使模型获得外部记忆, 因此可以利用特定领域的 先验知识提高学习效率和实现小规模数据集的快速学 习ํำ

可解释的机器学习模型能够为有机化学领域机制 与规律的认识提供启发与补充. 如何构建机制明晰的 “白箱” 模型将是未来一个重要的研究方向. 通过机器学 习模型的建立再反馈于有机化学的认识, 未来机器学习 与有机化学研究将是互相促进、互相依存的关系.

\section{4 总结}

总的来说, 机器学习(特别是深度学习技术)已在有 机化学研究中获得了初步的应用, 可以进行分子从头设 计、提出切合实际的合成路线、预测给定反应的产物和 产率、开发新的选择性催化剂、优化反应条件, 并应用 于自动化平台中. 现阶段, 为有效地使用机器学习来促 进研究进展, 在有机化学领域开展机器学习的使用教学 工作是十分必要的. 此外, 如何开发开放的大型数据 库、获取高质量和标准化的数据、更有效的表示分子和 反应、运用和开发适合研究有机化学领域的机器学习算 法以及建立有效、通用的算法评价基准, 将是未来机器 学习在有机化学领域应用的重要议题. 未来机器学习在 有机化学研究中的应用会持续增加, 有机化学工作者有 必要了解模型背后的理论框架, 在机器学习中引入物理 有机的策略和理念, 发展有化学意义的分子描述, 用物 理有机的语言连接量子力学和数学算法, 变黑箱模型为 白箱模型. 可以预期, 以深度机器学习为代表的人工智 能技术的引入和贯通应用, 将为有机化学研究的范式变 革提供诸多可能.

\section{References}

[1] McCarthy, J.; Minsky, M. L.; Rochester, N.; Shannon, C. E. A Proposal for the Dartmouth Summer Research Project on Artificial Intelligence, August 31, 1955, AI Magazine, 2006, 27, 12.

[2] Jordan, M. I.; Mitchell, T. M. Science 2015, 349, 255.

[3] Silver, D.; Huang, A.; Maddison, C.; Guez, A.; Sifre, L.; van den Driessche, G.; Schrittwieser, J.; Antonoglou, I.; Panneershelvam, V.; Lanctot, M.; Dieleman, S.; Grewe, D.; Nham, J.; Kalchbrenner, N.; Sutskever, I.; Lillicrap, T.; Leach, M.; Kavukcuoglu, K.; Graepel, T.; Hassabis, D. Nature 2016, 529, 484.

[4] Skoraczyński, G.; Dittwald, P.; Miasojedow, B.; Szymkuć, S.; Gajewska, E. P.; Grzybowski, B. A.; Gambin, A. Sci. Rep. 2017, 7 ,
3582.

[5] Samuel, A. L. IBM J. Res. Dev. 1959, 3, 210.

[6] Tenenbaum, J. B.; Kemp, C.; Griffiths, T. L.; Goodman, N. D. Science 2011, 331, 1279.

[7] (a) Rupp, M. Phys. Rev. Lett. 2012, 108, 058301. (b) Müller, K.-R. J. Chem. Theory Comput. 2013, 9, 3404

[8] Brockherde, F.; Vogt, L.; Li, L.; Tuckerman, M. K.; Burke, K.; Müller, K.-R. Nat. Commun. 2017, 8, 872.

[9] (a) Stokes, J. M.; Yang, K.; Swanson, K.; Jin, W.; Cubillos-Ruiz, A.; Donghia, N. M.; MacNair, C. R.; French, S.; Carfrae, L. A.; Bloom-Ackerman, Z.; Tran, V. M.; Chiappino-Pepe, A.; Badran, A. H.; Andrews, L. W.; Chory, E. J.; Church, G. M.; Brown, E. D.; Jaakkola, T. S.; Barzilay, R.; Collins, J. J. Cell 2020, 180, 688.

(b) Li, W.; Yang, J. C.; Huang, N. Acta Pharm. Sin. 2019, 54, 761 (in Chinese) (李伟，杨金才，黄牛，药学学报, 2019, 54, 761.)

[10] (a) Sun, T. L.; Pei, J. F. Chin. Sci. Bull. 2015, 60, 689 (in Chinese). (孙潭霖，裴剑锋，科学通报, 2015, 60, 689.)

(b) Gómez-Bombarelli, R.; Wei, J. N.; Duvenaud, D.; Hernández-Lobato, J. M.; Sánchez-Lengeling, B.; Sheberla, D.; AguileraIparraguirre, J.; Hirzel, T. D.; Adams, R. P.; Aspuru-Guzik, A. ACS Cent. Sci. 2018, 4, 268

(c) Segler, M. H. S.; Kogej, T.; Tyrchan, C.; Waller, M. P. ACS Cent. Sci. 2018, 4, 120 .

[11] (a) Segler, M. H. S.; Preuss, M.; Waller, M. P. Nature 2018, 555, 604.

(b) Segler, M. H. S.; Waller, M. P. Chem.-Eur. J. 2017, 23, 5966.

[12] Granda, J. M.; Donina, L.; Dragone, V.; Long, D.-L.; Cronin, L. Nature 2018, 559, 377.

[13] Warr, W. A. Mol. Inf. 2014, 33, 469.

[14] Blum, L. C.; Reymond, J.-L. J. Am. Chem. Soc. 2009, 131, 8732.

[15] Ruddigkeit, L.; van Deursen, R.; Blum, L. C.; Reymond, J.-L. J. Chem. Inf. Model. 2012, 52, 2864.

[16] Delaney, J. S. J. Chem. Inf. Comput. Sci. 2004, 44, 1000.

[17] Mobley, D. L.; Guthrie, J. P. J. Comput.-Aided Mol. Des. 2014, 28, 711.

[18] Sterling, T.; Irwin, J. J. J. Chem. Inf. Model. 2015, 55, 2324

[19] (a) Akhondi, S. A.; Klenner, A. G.; Tyrchan, C.; Manchala, A. K.; Boppana, K.; Lowe, D.; Zimmermann, M.; Jagarlapudi, S. A. R. P.; Sayle, R.; Kors, J. A.; Muresan, S. PloS One 2014, 9, el07477.

(b) Southan, C. Drug Discovery Today: Technol. 2015, 14, 3.

(c) Lowe, D. M. PhD. Dissertation, University of Cambridge, Cambridge, 2012.

[20] Manickam, Y.; Chaturvedi, R.; Babbar, P.; Malhotra, N.; Jain, V.; Sharma, A. Drug Discovery Today 2018, 23, 6.

[21] (a) Schneider, N.; Lowe, D. M.; Sayle, R. A.; Landrum, G. A. J. Chem. Inf. Model. 2015, 55, 39.

(b) Schneider, N.; Lowe, D. M.; Sayle, R. A.; Tarselli, M. A.; Landrum, G. A. J. Med. Chem. 2016, 59, 4385.

[22] Rahman, S. A.; Torrance, G.; Baldacci, L.; Cuesta, S. M.; Fenninger, F.; Gopal, N.; Choudhary, S.; May, J. W.; Holliday, G. L.; Steinbeck, C.; Thornton, J. M. Bioinformatics 2016, 32, 2065.

[23] Cooper, T. W. J.; Campbell, I. B.; Macdonald, S. J. F. Angew. Chem., Int. Ed. 2010, 49, 8082.

[24] Buitrago Santanilla, A.; Regalado, E. L.; Pereira, T.; Shevlin, M.; Bateman, K.; Campeau, L.-C.; Schneeweis, J.; Berritt, S.; Shi, Z.-C.; Nantermet, P.; Liu, Y.; Helmy, R.; Welch, C. J.; Vachal, P.; Davies, I. W.; Cernak, T.; Dreher, S. D. Science 2015, 347, 49

[25] Tetko, I. V.; Engkvist, O.; Chen, H. Future Med. Chem. 2016, 8, 1801.

[26] ChemAxon http://chemaxon.com.

[27] Landrum, G. RDKit: Open-source Cheminformatics, 2014, http://www. rdkit.org.

[28] (a) Steinbeck, C.; Han, Y.; Kuhn, S.; Horlacher, O.; Luttmann, E.; Willighagen, E. J. Chem. Inf. Comput. Sci. 2003, 43, 493. (b) Steinbeck, C.; Hoppe, C.; Kuhn, S.; Floris, M.; Guha, R.; Willighagen, E. L. Curr. Pharm. Des. 2006, 12, 2111.

(c) Chemistry Development Kit, 2014, https://cdk.github.io/. 
[29] Durant, J. L.; Leland, B. A.; Henry, D. R.; Nourse, J. G. J. Chem. Inf. Comput. Sci. 2002, 42, 1273.

[30] (a) Cereto-Massague, A.; Jose Ojeda, M.; Valls, C.; Mulero, M.; Garcia-Vallve, S.; Pujadas, G. Methods 2015, 71, 58.

(b) Muegge, I.; Mukherjee, P. Expert Opin. Drug Discovery 2016, 11,137 .

[31] (a) Bender, A.; Mussa, H. Y.; Glen, R. C.; Reiling, S. J. Chem. Inf. Comput. Sci. 2004, 44, 170.

(b) Bender, A.; Mussa, H. Y.; Glen, R. C.; Reiling, S. J. Chem. Inf. Comput. Sci. 2004, 44, 1708.

[32] Morgan, H. L. J. Chem. Doc. 1965, 5, 107.

[33] Rogers, D.; Hahn, M. J. Chem. Inf. Model. 2010, 50, 742.

[34] O'Boyle, N. M.; Banck, M.; James, C. A.; Morley, C.; Vandermeersch, T.; Hutchison, G. R. J. Cheminf. 2011, 3, 33.

[35] Indigo-GGA Software Services 2014. https://github.com/ggasoftware/indigo.

[36] Weininger, D. J. Chem. Inf. Comput. Sci. 1988, $28,31$.

[37] Heller, S.; McNaught, A.; Stein, S.; Tchekhovskoi, D.; Pletnev, I. J. Cheminf. 2013, 5,7 .

[38] Jeliazkova, N.; Kochev, N. Mol. Inf. 2011, 30, 707.

[39] Raymond, J. W.; Willett, P. J. Comput.-Aided Mol. Des. 2002, 16, 521.

[40] Rupp, M.; Tkatchenko, A.; Muller, K. R.; von Lilienfeld, O. A. Phys. Rev. Lett. 2012, 108, 058301.

[41] (a) Hochuli, J.; Helbling, A.; Skaist, T.; Ragoza, M.; Koes, D. R. J. Mol. Graphics Modell. 2018, 84, 96.

(b) Ragoza, M.; Hochuli, J.; Idrobo, E.; Sunseri, J.; Koes, D. R. J. Chem. Inf. Model. 2017, 57, 942.

[42] Zahrt, A. F.; Henle, J. J.; Rose, B. T.; Wang, Y.; Darrow, W. T.; Denmark, S. E. Science 2019, 363, eaau5631.

[43] (a) Sutton, R. S. Mach. Learn. 1988, 3, 9.

(b) François-Lavet, V.; Henderson, P.; Islam, R.; Bellemare, M. G.; Pineau, J. Found. Trends Mach. Learn. 2018, 11, 219.

[44] (a) Koski, T.; Noble, J. Mathematica Applicanda (Matematyka Stosowana) 2012, 40, 51

(b) Spiegelhalter, D. J. J. R. Statist. Soc. C 1998, 47, 115.

[45] Quinlan, J. R. Mach. Learn. 1986, 1, 81.

[46] (a) Domingos, P.; Pazzani, M. Mach. Learn. 1997, 29, 103.

(b) Webb, G. I.; Boughton, J. R.; Wang, Z. Mach. Learn. 2005, 58,

(c) Maron, M. E. J. ACM 1961, 8, 404.

[47] Cortes, C.; Vapnik, V. Mach. Learn. 1995, 20, 273

[48] (a) Achtert, E.; Böhm, C.; Kriegel, H.-P.; Kröger, P.; M ü ller-Gorman, I.; Zimek, A. In Finding Hierarchies of Subspace Clusters, Knowledge Discovery in Databases: PKDD 2006 Series 4213, Springer Berlin Heidelberg, Heidelberg, 2006, pp. 446 453. (b) Kriegel, H.-P.; Kröger, P.; Zimek, A. WIREs Data Mining Knowl. Discov. 2012, 2, 351.

(c) Sibson, R. Comput. J. 1973, 16, 30.

(d) Banerjee, A.; Dave, R. N. In Validating Clusters using the Hopkins Statistic, 2004 IEEE International Conference on Fuzzy Systems (IEEE Cat. No. 04CH37542), IEEE, Budapest, 2004, pp. $149 \sim 153$.

(e) Estivill-Castro, V. SIGKDD Explor. Newsl. 2002, 4, 65.

[49] (a) Breiman, L. Mach. Learn. 2001, 45, 5.

(b) Ho, T. K. IEEE Trans. Pattern Anal. Mach. Intell. 1998, 20, 832.

[50] Zhou, Z. H. Machine Learning, Tsinghua University Press, Beijing, 2016 (in Chinese) (周志华, 机器学习, 清华大学出版社, 北京, 2016.)

[51] Pedregosa, F.; Varoquaux, G.; Gramfort, V.; Michel, B.; Thirion, O.; Grisel, M.; Blondel, P.; Prettenhofer, R.; Weiss, V.; Dubourg, V.; Vanderplas, J.; Passos, A.; Cournapeau, D.; Brucher, M.; Perrot, M.; Duchesnay, E. J. Mach. Learn. Res. 2011, 12, 2825.

[52] (a) Maass, W. Neural Net. 1997, 10, 1659.

(b) Wang, W.; Pedretti, G.; Milo, V.; Carboni, R.; Calderoni, A.; Ramaswamy, N.; Spinelli, A. S.; Ielmini, D. Sci. Adv. 2018, 4, eaat 4752 . (c) Tavanaei, A.; Ghodrati, M.; Kheradpisheh, S. R.; Masquelier, T.; Maida, A. Neural Netw. 2019, 111, 47.

[53] McCulloch, W. S.; Pitts, W. Bull Math. Biophys. 1943, 5, 115.

[54] Srivastava, N.; Hinton, G.; Krizhevsky, A.; Sutskever, I.; Salakhutdinov, R. J. Mach. Learn. Res. 2014, 15, 1929.

[55] Li, W.; Matthew, Z.; Sixin, Z.; Yann Le, C.; Rob, F. In Proceedings of the 30th International Conference on Machine Learning, Proceedings of Machine Learning Research Series 28, Eds.: Dasgupta, S.; McAllester, D., Proceedings of Machine Learning Research, Atlanta, 2013, pp. 1058 1066

[56] Nair, V.; Hinton, G. E. In International Conference on Machine Learning, Proceedings of the 27th International Conference on Machine Learning, International Conference on Machine Learning Series 27, International Conference on Machine Learning, Haifa, 2010.

[57] (a) Zhou, J.; Cui, G.; Zhang, Z. Y.; Yang, C.; Liu, Z. Y.; Wang, L. F.; Li, C. C.; Sun, M. arXiv e-prints 2018, arXiv: 1812.08434.

(b) Zhang, Z. W.; Cui, P.; Zhu, W. W. IEEE Trans. Knowl. Data Eng. 2020, doi:10.1109/TKDE.2020.2981333.

[58] (a) Duvenaud, D.; Maclaurin, D.; Aguilera-Iparraguirre, J.; Gómez-Bombarelli, R.; Hirzel, T.; Aspuru-Guzik, A.; Adams, R. P. In Advances in Neural Information Processing Systems 28, Neural Information Processing Systems 2015, Eds.: Cortes, C.; Lawrence, N.; Lee D.; Sugiyama, M.; Garnett, R., Neural Information Processing Systems, 2015, pp. 2215 2223.

(b) Coley, C. W.; Jin, W.; Rogers, L.; Jamison, T. F.; Jaakkola, T. S.; Green, W. H.; Barzilay, R.; Jensen, K. F. Chem. Sci. 2019, 10, 370.

[59] Mitchell, J. B. O. Wires Comput. Mol. Sci. 2014, 4, 468.

[60] Corey, E. J.; Wipke, W. T. Science 1969, 166, 178.

[61] Muratov, E. N.; Bajorath, J.; Sheridan, R. P.; Tetko, I. V.; Filimonov, D.; Poroikov, V.; Oprea, T. I.; Baskin, I. I.; Varnek, A.; Roitberg, A.; Isayev, O.; Curtalolo, S.; Fourches, D.; Cohen, Y.; Aspuru-Guzik, A.; Winkler, D. A.; Agrafiotis, D.; Cherkasov, A.; Tropsha, A. Chem. Soc. Rev. 2020, 49, 3525.

[62] Ma, J.; Sheridan, R. P.; Liaw, A.; Dahl, G. E.; Svetnik, V. J. Chem. Inf. Model. 2015, 55, 263.

[63] Mayr, A.; Klambauer, G.; Unterthiner, T.; Hochreiter, S. Front. Environ. Sci. 2016, 3, 80

[64] (a) Ramsundar, B.; Liu, B.; Wu, Z.; Verras, A.; Tudor, M.; Sheridan, R. P.; Pande, V. J. Chem. Inf. Model. 2017, 57, 2068.

(b) Koutsoukas, A.; Monaghan, K. J.; Li, X.; Huan, J. J. Cheminf. 2017, 9, 42 .

(c) Lenselink, E. B.; ten Dijke, N.; Bongers, B.; Papadatos, G.; van Vlijmen, H. W. T.; Kowalczyk, W.; Ijzerman, A. P.; van Westen, G. J. P. J. Cheminf. 2017, 9, 45.

[65] (a) Subramanian, G.; Ramsundar, B.; Pande, V.; Denny, R. A. J. Chem. Inf. Model. 2016, 56, 1936.

(b) Aliper, A.; Plis, S.; Artemov, A.; Ulloa, A.; Mamoshina, P.; Zhavoronkov, A. Mol. Pharm. 2016, 13, 2524.

(c) Lusci, A.; Pollastri, G.; Baldi, P. J. Chem. Inf. Model. 2013, 53, 1563.

(d) Xu, Y.; Dai, Z.; Chen, F.; Gao, S.; Pei, J.; Lai, L. J. Chem. Inf. Model. 2015, 55, 208.

[66] Ryu, S.; Kwon, Y.; Kim, W. Y. Chem. Sci. 2019, 10, 8438.

[67] Gaulton, A.; Hersey, A.; Nowotka, M.; Patrícia Bento, A.; Chambers, J.; Mendez, D.; Mutowo, P.; Atkinson, F.; Bellis, L. J.; Cibrián-Uhalte, E.; Davies, M.; Dedman, N.; Karlsson, A.; Magariños, M. P.; Overington, J. P.; Papadatos, G.; Smit, I.; Leach, A. L. Nucleic Acids Res. 2017, 45, 945.

[68] (a) Fraczkiewicz, R.; Lobell, M.; Göller, A. H.; Krenz, U.; Schoenneis, R.; Clark, R. D.; Hillisch. A. J. Chem. Inf. Model. $\mathbf{2 0 1 5}, 55,389$.

(b) Fraczkiewicz, R.; Lobell, M.; Göller, A. H.; Krenz, U.; Schoenneis, R.; Clark, R. D.; Hillisch, A. J. Chem. Inf. Model. 2015, 55, 389 .

[69] Roszak, R.; Beker, W.; Molga, K.; Grzybowski, B. A. J. Am. Chem. Soc. 2019, 141, 17142.

[70] Yang, Q.; Li, Y.; Yang, J.-D.; Liu, Y. D.; Zhang, L.; Luo, S. Z.; 
Cheng, J.-P. Angew. Chem., Int. Ed. 2020, 59, 19282.

[71] Yang, J.-D.; Xue, X.-S.; Ji, P.; Li, X.; Cheng, J.-P. Internet Bond-energy Databank ( $p K_{a}$ and BDE): iBonD Home Page, http: //ibond.chem.tsinghua.edu.cn or http://ibond.nankai.edu.cn.

[72] Hall, L. H. J. Chem. Inf. Comput. Sci. 1995, 35, 1039.

[73] Feng, C.; Sharman, E.; Ye, S.; Luo, Y.; Jiang, J. Sci. China: Chem. 2019, 62, 1698

[74] Gilmer, J.; Schoenholz, S. S.; Riley, P. F.; Vinyals, O.; Dahl, G. E. Proceedings of the 34th International Conference on Machine Learning, In Proceedings of Machine Learning Research Series 70, Eds.: Precup, D.; Teh, Y. W., Proceedings of Machine Learning Research, Sydney, 2017, pp. 1263 1272.

[75] Ertl, P.; Lewis, R.; Martin, E.; Polyakov, V. arXiv e-prints 2017, arXiv: 1712.07449 .

[76] Liang, L.; Deng, C. L.; Zhang, Y. M.; Hua, Y.; Liu, H. C.; Lu, T.; Chen, Y. D. Prog. Pharm. Sci. 2020, 44, 18 (in Chinese). (梁礼, 邓成龙, 张艳敏, 滑艺, 刘海春, 陆涛, 陈亚东, 药学进 展, 2020, 44, 18.)

[77] Kadurin, A.; Nikolenko, S.; Khrabrov, K.; Aliper, A.; Zhavoronkov, A. Mol. Pharm. 2017, 14, 3098.

[78] Goodfellow, I. arXiv e-prints 2016, arXiv: 1701.00160

[79] Zhavoronkov, A.; Ivanenkov, Y. A.; Aliper, A.; Veselov, M. S.; Aladinskiy, V. A.; Aladinskaya, A. V.; Terentiev, V. A.; Polykovskiy, D. A.; Kuznetsov, M. D.; Asadulaev, A.; Volkov, Y.; Zholus, A.; Shayakhmetov, R. R.; Zhebrak, A.; Minaeva, L. I.; Zagribelnyy, B. A.; Lee, L. H.; Soll, R.; Madge, D.; Xing, L.; Guo, T.; Aspuru-Guzik, A. Nat Biotechnol. 2019, 37, 1038 .

[80] Yu, L. T.; Zhang, W. N.; Wang, J.; Yu, Y. arXiv e-prints 2016, arXiv: 1609.05473 .

[81] Jaques, N.; Gu, S.; Bahdanau, D.; Hernández-Lobato, J. M.; Turner, R. E.; Eck, D. arXiv e-prints 2016, arXiv: 1611. 02796.

[82] Leo, A.; Hansch, C.; Elkins, D. Chem. Rev. 1971, 71, 525.

[83] Bickerton, G. R.; Paolini, G. V.; Besnard, J.; Muresan, S.; Hopkins, A. L. Nat. Chem. 2012, 4, 90.

[84] Olivecrona, M.; Blaschke, T.; Engkvist, O.; Chen, H. J. Cheminf. 2017, 9, 48 .

[85] Benhenda, M. arXiv e-prints 2017, arXiv: 1708.08227.

[86] (a) Metz, L.; Poole, B.; Pfau, D.; Sohl-Dickstein, J. arXiv e-prints 2016, arXiv: 1611.02163 .

(b) Unterthiner, T.; Nessler, B.; Seward, C.; Klambauer, G.; Heusel, M.; Ramsauer, H.; Hochreiter, S. arXiv e-prints 2017, arXiv: 1708.08819

[87] Corey, E. J.; Wipke, W. T.; Cramer, R. D.; Howe, W. J. J. Am. Chem. Soc. 1972, 94, 431

[88] Hendrickson, J. B. Recl. Trav. Chim. Pays-Bas. 1992, 111, 323.

[89] (a) Todd, M. H. Chem. Soc. Rev. 2005, 34, 247.

(b) Szymkuć, S.; Gajewska, E. P.; Klucznik, T.; Molga, K.; Dittwald, P.; Startek, M.; Bajczyk, M.; Grzybowski, B. A. Angew. Chem., Int. Ed. 2016, 55, 5904.

[90] de Almeida, A. F.; Moreira, R.; Rodrigues, T. Nat. Rev. Chem. 2019, 3, 589 .

[91] Kayala, M. A.; Azencott, C.-A.; Chen, J. H.; Baldi, P. J. Chem. Inf. Model. 2011, 51, 2209.

[92] Kayala, M. A.; Baldi, P. J. Chem. Inf. Model. 2012, 52, 2526.

[93] Schwaller, P.; Laino, T.; Gaudin, T.; Bolgar, P.; Hunter, C. A.; Bekas, C.; Lee, A. A. ACS Cent. Sci. 2019, 5, 1572.

[94] McDaniel, D. H.; Brown, H. C. J. Org. Chem. 1958, 23, 420.

[95] (a) Friedman, M.; Wall, J. S. J. Am. Chem. Soc. 1964, 86, 3735. (b) Friedman, M.; Cavins, J. F.; Wall, J. S. J. Am. Chem. Soc. 1965, 87,3672 .

(c) Friedman, M.; Wall, J. S. J. Org. Chem. 1966, 31, 2888.

[96] Toropov, A. A.; Kudyshkin, V. O.; Voropaeva, N. L.; Ruban, I. N.; Rashidova, S. S. J. Struct. Chem. 2004, 45, 945.

[97] Yu, X.; Yi, B.; Wang, X. Eur. Polym. J. 2008, 44, 3997.

[98] Morrill, J. A.; Biggs, J. H.; Bowman, C. N.; Stansbury, J. W. J. Mol. Graphics Modell. 2011, 29, 763 .

[99] Schwöbel, J. A. H.; Wondrousch, D.; Koleva, Y. K.; Madden, J. C.; Cronin, M. T. D.; Schüürmann, G. Chem. Res. Toxicol. 2010, 23,
1576.

[100] Wondrousch, D.; Böhme, A.; Thaens, D.; Ost, N.; Schüürmann, G. J. Phys. Chem. Lett. 2010, 1, 1605.

[101] (a) Zhang, L.; Li, X.; Luo, S. Z.; Cheng, J.-P. Sci. Sin.: Chim. 2016 46, 535 (in Chinese).

(张龙, 李金金, 罗三中, 程津培, 中国科学: 化学, 2016, 46, 535.)

(b) Li, Y.; Luo, S. Z. Chin. J. Org. Chem. 2018, 38, 2363 (in Chinese).

(李遥, 罗三中, 有机化学, 2018, 38, 2363.)

[102] Halberstam, N. M.; Baskin, I. I.; Palyulin, V. A.; Zefirov, N. S. Mendeleev Commun. 2002, 12, 185.

[103] Harper, K. C.; Bess, E. N.; Sigman, M. S. Nat. Chem. 2012, 4, 366.

[104] Ahneman, D. T.; Estrada, J. G.; Lin, S.; Dreher, S. D.; Doyle, A. G. Science 2018, 360, 186.

[105] Singh, S.; Pareek, M.; Changotra, A.; Banerjee, S.; Bhaskararao, B.; Balamurugan, P.; Sunoj, R. B. Proc. Natl. Acad. Sci. U. S. A. 2020, $117,1339$.

[106] Li, X.; Zhang, S.-Q.; Xu, L.-C.; Hong. X. Angew. Chem., Int. Ed. 2020, 59, 13253.

[107] Sandfort, F.; Strieth-Kalthoff, F.; Kuhnemund, M.; Beecks, C.; Glorius, F. Chem $\mathbf{2 0 2 0}, 6,1$.

[108] Lin, A. I.; Madzhidov, T. I.; Klimchuk, O.; Nugmanov, R. I.; Antipin, I. S.; Varnek, A. J. Chem. Inf. Model. 2016, 56, 2140.

[109] Marcou, G.; Aires, de Sousa. J.; Latino, D. A. R. S.; de Luca, A.; Horvath, D.; Rietsch, V.; Varnek, A. J. Chem Inf. Model. 2015, 55, 239.

[110] Gao, H.; Struble, T. J.; Coley, C. W.; Wang, Y.; Green, W. H.; Jensen, K. F. ACS Cent. Sci. 2018, 4, 1465.

[111] Struebing, H.; Ganase, Z.; Karamertzanis, P.; Siougkrou, E.; Haycock, P.; Piccione, P. M.; Armstrong, A.; Galindo, A.; Adjiman, C. S. Nat. Chem. 2013, 5, 952 .

[112] Häse, F.; Roch, L. M.; Kreisbeck, C.; Aspuru-Guzik, A. ACS Cent. Sci. 2018, 4, 1134.

[113] (a) Baskin, I. I.; Madzhidov, T. I.; Antipin, I. S.; Varnek, A. A. Russ. Chem. Rev. 2017, 86, 1127

(b) Cook, A.; Johnson, A. P.; Law, J.; Mirzazadeh, M.; Ravitz, O.; Simon, A. Science 2012, 2, 79

(c) Zefirov, N. S.; Gordeeva, E. V. Russ. Chem. Rev. 1987, 56, 1002.

[114] Coley, C. W.; Green, W. H.; Jensen, K. F. Acc. Chem. Res. 2018, 51 , 1281.

[115] Varnek, V.; Baskin, I. I. In Systems Medicine, Vol. 2, Eds.: Wolkenhauer, O., Academic Press, Oxford, 2021, pp. 190 197.

[116] Law, J.; Zsoldos, Z.; Simon, A.; Reid, D.; Liu, Y.; Khew, S. Y.; Johnson, A. P.; Major, S.; Wade, R. A.; Ando, H. Y. J. Chem. Inf. Model. 2019, 49, 593.

[117] Coley, C. W.; Rogers, L.; Green, W. H.; Jensen, K. F. ACS Cent. Sci. 2017, 3, 1237.

[118] Liu, B.; Ramsundar, B.; Kawthekar, P.; Shi, J.; Gomes, J.; Luu Nguyen, Q.; Ho, S.; Sloane, J.; Wender, P.; Pande, V. ACS Cent. Sci. 2017, 3, 1103 .

[119] Lin, L. J.; Xu, Y. J.; Pei, J. F.; Lai, L, H. Chem. Sci. 2020, 11, 3355.

[120] Schwaller, P.; Petraglia, R.; Zullo, V.; Nair, V. H.; Haeuselmann, R. A.; Pisoni, R.; Bekas, C.; Iuliano, A.; Laino, T. Chem. Sci. 2020, 11, 3316.

[121] (a) Segler, M. H. S.; Preuss, M.; Waller, M. P. Nature 2018, 555 , 604.

(b) Satoh, H.; Funatsu, K. J. Chem. Inf. Comput. Sci. 1995, 35, 34.

[122] Schwaller, P.; Vaucher, A.; Nair, V. H.; Laino, T.; Reymond, J.-L. ChemRxiv Preprint 2019, https://doi.org/10.26434/chemrxiv. 9897365.v2.

[123] (a) Trobe, M.; Burke, M. D. Angew. Chem., Int. Ed. 2018, 57, 4192. (b) Ley, S. V.; Fitzpatrick, D. E.; Ingham, R. J.; Myers, R. M. Angew. Chem., Int. Ed. 2015, 54, 3449.

[124] Sans, V.; Cronin, L. Chem. Soc. Rev. 2016, 45, 2032.

[125] Houben, C.; Lapkin, A. A. Curr. Opin. Chem. Eng. 2015, 9, 1.

[126] Perera, D.; Tucker, J. W.; Brahmbhatt, S.; Helal, C. J.; Chong, A.; Farrell, W.; Richardson, P.; Sach, N. W. Science 2018, 359, 429. 
[127] Cortés-Borda, D.; Wimmer, E.; Gouilleux, B.; Barré, E.; Oger, N.; Goulamaly, L.; Peault, L.; Charrier, B.; Truchet, C.; Giraudeau, P.; Rodriguez-Zubiri, M.; Le Grognec, E.; Felpin, F.-X. J. Org. Chem. 2018, 83, 14286.

[128] Steiner, S.; Wolf, J.; Glatze, S.; Andreou, A.; Granda, J. M.; Keenan, G.; Hinkley, T.; Aragon-Camarasa, G.; Kitson, P. J.; Angelone, D.; Cronin, L. Science 2019, 363, eaav2211.

[129] Coley, C. W.; Thomas D. A.; Lummiss, J. A. M.; Jaworski, J. N.; Breen, C. P.; Schultz, V.; Hart, T.; Fishman, J. S.; Rogers, L.; Gao, H.; Hicklin, R. W.; Plehiers, P. P.; Byington, J.; Piotti, J. S.; Green, W. H.; Hart, A. J.; Jamison, T. F.; Jensen, K, F. Science 2019, 365, eaax 1566 .

[130] Chatterjee, S.; Guidi, M.; Seeberger, P. H.; Gilmore. K. Nature 2020, 579, 379.

[131] (a) Mysinger, M. M.; Carchia, M.; Irwin, J. J.; Shoichet, B. K. J. Med. Chem. 2012, 55, 6582 .

(b) Sun, J.; Jeliazkova, N.; Chupakhin, V.; Golib-Dzib, J.-F.; Engkvist, O.; Carlsson, L.; Wegner, J.; Ceulemans, H.; Georgiev, I.; Jeliazkov, V.; Kochev, N.; Ashby, T. J.; Chen, H. J. Cheminf. 2017, 9, 17.

[132] Russakovsky, O.; Deng, J.; Su, H.; Krause, J.; Satheesh, S.; Ma, S.; Huang, Z.; Karpathy, A.; Khosla, A.; Bernstein, M.; Berg, A. C.; Li, F.-F. Int. J. Comput. Vision 2015, 115, 211.

[133] Miller, G. A. Commun. ACM 1995, 38, 39.

[134] Wu, Z.; Ramsundar, B.; Feinberg, Evan N.; Gomes, J.; Geniesse, C.; Pappu, A. S.; Leswing, K.; Pande, V. Chem. Sci. 2018, 9, 513.

[135] Kitchin, J. R. Nat. Cat. 2018, 1, 230.

[136] Raccuglia, P.; Elbert, K. C.; Adler, P. D. F.; Falk, C.; Wenny, M. B.; Mollo, A.; Zeller, M.; Friedler, S. A.; Schrier, J.; Norquist, A. J. Nature 2016, 533, 73.

[137] Struble, T. J.; Alvarez, J. C.; Brown, S. P.; Chytil, M.; Cisar, J.; DesJarlais, R. L.; Engkvist, O,; Frank, S. A.; Greve, D. R.; Griffin, D. J.; Hou, X. J.; Johannes, J. W.; Kreatsoulas, C.; Lahue, B.; Mathea, M.; Mogk, G.; Nicolaou, C. A.; Palmer, A. D.; Price, D. J.; Robinson, R. I.; Salentin, S.; Xing, L.; Jaakkola, T.; Green, W. H.; Barzilay, R.; Coley, C. W.; Jensen, K. F. J. Med. Chem. 2020, 63, 8667.

[138] Caramelli, D.; Salley, D.; Henson, A.; Camarasa, G. A.; Sharabi, S.; Keenan, G.; Cronin, L. Nat. Commun. 2018, 9, 3406.
[139] (a) Goodell, J. R.; McMullen, J. P.; Zaborenko, N.; Maloney, J. R.; Ho, C.-X.; Jensen, K. F.; Porco, J. A. Jr.; Beeler, A. B. J. Org. Chem. 2009, 74, 6169.

(b) Heublein, N.; Moore, J. S.; Smith, C. D.; Jensen, K. F. RSV Adv. 2014, 4, 63627.

(c) Weber, A.; von Roedern, E.; Stilz, H. U. J. Comb. Chem. 2005, 7, 178.

[140] Duvenaud, D.; Maclaurin, D.; Aguilera-Iparraguirre, J.; Gómez-Bombarelli, R.; Hirzel, T.; Aspuru-Guzik, A.; Adams, A. P. In Advances in Neural Information Processing Systems 28, Neural Information Processing Systems 2015, Eds.: Cortes, C.; Lawrence, N.; Lee D.; Sugiyama, M.; Garnett, R., Neural Information Processing Systems, 2015, pp. 2224 2232 .

[141] Jankowski, N., Duch, W.; Grạbzewski, K. Meta-Learning in Computational Intelligence, Springer, Berlin, 2011.

[142] Graves, A.; Wayne, G.; Danihelka, I. arXiv e-prints 2014, arXiv: 1410. 5401.

[143] Duan, Y.; Andrychowicz, M.; Stadie, B. C.; Ho, J.; Schneider, J.; Sutskever, I.; Abbeel, P.; Zaremba W. In Advances in Neural Information Processing Systems 30, Neural Information Processing Systems 2017, Eds.: Guyon, I.; Luxburg, U. V.; Bengio, S.; Wallach, H.; Fergus, R.; Vishwanathan, S.; Garnett, R., Neural Information Processing Systems, 2017, pp. 1087 1098 .

[144] Lake, B. M.; Salakhutdinov, R.; Tenenbaum, J. B. Science 2015, $350,1332$.

[145] Vinyals, O.; Blundell, C.; Lillicrap, T.; Kavukcuoglu, K.; Wierstra, D. In Advances in Neural Information Processing Systems 29, Neural Information Processing Systems 2016, Eds.: Lee, D.; Sugiyama, M.; Luxburg, U.; Guyon, I.; Garnett, R., Neural Information Processing Systems, 2016, pp. 3630 3638 .

[146] Altae-Tran, H.; Ramsundar, B.; Pappu, A. S.; Pande, V. ACS Cent. Sci. 2017, 3, 283.

[147] (a) Santoro, A.; Bartunov, S.; Botvinick, M.; Wierstra, D.; Lillicrap T. In Proceedings of The 33rd International Conference on Machine Learning, Proceedings of Machine Learning Research Series 48, Eds.: Balcan, M. F.; Weinberger, K. Q., Proceedings of Machine Learning Research, New York, 2016, pp. 1842 1850 .

(b) Ha, H.; Hwang, U.; Hong, Y.; Yoon, S. arXiv e-prints 2018. arXiv: 1805.10768 .

(Zhao, C.) 